\title{
The radio properties of radio-loud narrow-line Seyfert 1 galaxies on parsec scales
}

\author{
Minfeng $\mathrm{Gu}^{1,2}$, Yongjun Chen ${ }^{1,3}$, S. Komossa ${ }^{4}$, Weimin Yuan ${ }^{5}$, Zhiqiang Shen ${ }^{1,3}$, Kiyoaki \\ Wajima $^{6}$, Hongyan Zhou ${ }^{7,8}$, J. A. Zensus ${ }^{4}$
}

\begin{abstract}
We present the detection of compact radio structures of fourteen radio-loud narrow line Seyfert 1 (NLS1) galaxies from Very Long Baseline Array observations at $5 \mathrm{GHz}$, which were performed in 2013 . While $50 \%$ of the sources of our sample show a compact core only, the remaining $50 \%$ exhibit a core-jet structure. The measured brightness temperatures of the cores range from $10^{8.4}$ to $10^{11.4} \mathrm{~K}$ with a median value of $10^{10.1} \mathrm{~K}$, indicating that the radio emission is from non-thermal jets, and that, likely, most sources are not strongly beamed, then implying a low jet speed in these radio-loud NLS1 galaxies. In combination with archival data taken at multiple frequencies, we find that seven sources show flat or even inverted radio spectra, while steep spectra are revealed in the remaining seven objects. Although all these sources are very radio-loud with $R>100$, their jet properties are diverse, in terms of their milli-arcsecond (mas) scale (pc scale) morphology and their overall radio spectral shape. The evidence for slow jet speeds (i.e., less relativistic jets), in combination with the low kinetic/radio power, may offer an
\end{abstract}

\footnotetext{
${ }^{1}$ Shanghai Astronomical Observatory, Chinese Academy of Sciences, Shanghai 200030, China; gumf@shao.ac.cn

${ }^{2}$ Key Laboratory for Research in Galaxies and Cosmology, Shanghai Astronomical Observatory, Chinese Academy of Sciences, 80 Nandan Road, Shanghai, 200030, China

${ }^{3}$ Key Laboratory of Radio Astronomy, Chinese Academy of Sciences, 2 West Beijing Road, Nanjing, JiangSu 210008, China

${ }^{4}$ Max-Planck-Institut für Radioastronomie, Auf dem Hügel 69, 53121 Bonn, Germany

${ }^{5}$ Key Lab for Space Astronomy and Technology, National Astronomical Observatories, Chinese Academy of Sciences, Beijing 100012, PR China

${ }^{6}$ Korea Astronomy and Space Science Institute, 776 Daedeokdae-ro, Yuseong, Daejeon 305-348, Korea

${ }^{7}$ Polar Research Institute of China, 451 Jinqiao Road, Shanghai, 200136, China

${ }^{8}$ Key Laboratory for Research in Galaxies and Cosmology, Department of Astronomy, University of Science and Technology of China, Chinese Academy of Sciences, Hefei, Anhui, 230026, China
} 
explanation for the compact VLBA radio structure in most sources. The mildly relativistic jets in these high accretion rate systems are consistent with a scenario, where jets are accelerated from the hot corona above the disk by the magnetic field and the radiation force of the accretion disk. Alternatively, a low jet bulk velocity can be explained by low spin in the Blandford-Znajek mechanism.

Subject headings: galaxies: active — galaxies: jets — galaxies: Seyfert — radio continuum: galaxies

\section{Introduction}

While both permitted and forbidden optical emission lines are present in narrow-line Seyfert 1 galaxies (NLS1s), their broad Balmer lines are narrower than those of normal broadline Seyfert 1 galaxies with FWHM(H $\beta$ ) less than $2000 \mathrm{~km} \mathrm{~s}^{-1}$ in NLS1s (e.g., Osterbrock \& Pogge 1985; Goodrich 1989). Moreover, NLS1s also exhibit other extreme observational properties, such as relatively weak forbidden-line emission, i.e., [OIII] $5007 / \mathrm{H} \beta<3$, strong permitted optical/UV Fe II emission lines, steep soft X-ray spectra, and rapid X-ray variability. NLS1s are often thought to be young AGNs with relatively small black hole masses and high accretion rates (review by Komossa 2008).

Conventionally, NLS1s were thought to be radio quiet. With the discovery of radio loud NLS1s (RLNLS1s), it has been realized, that NLS1s simply have a low probability to be radio loud, but are not completely radio quiet (Komossa et al. 2006; Zhou et al. 2006). Interestingly, RLNLS1s are inhomogeneous in their radio properties. As shown in Komossa et al. (2006), most RLNLS1s in their sample are compact, steep spectrum sources, and hence likely associated with compact steep-spectrum (CSS) radio sources. A few NLS1s, however, showed flat or inverted radio spectra, and other properties characteristic of blazars. In fact, further observational evidence has recently accumulated, that a significant fraction of RLNLS1s, especially at the highest radio-loudnesses, does display the characteristics of blazars, including large-amplitude radio flux and spectral variability, compact radio cores, very high variability brightness temperatures, enhanced optical continuum emission, flat X-ray spectra, and blazar-like spectral energy distributions (SED) (e.g., Yuan et al. 2008), and several of them have been detected in the $\gamma$-ray regime with Fermi for the first time (Abdo et al. 2009a, b).

Very long baseline interferometry (VLBI) is one of the most powerful tools for revealing the radio properties at parsec (pc) scales by direct imaging at milli-arcsecond resolution. The early Japanese VLBI Network observations of a few RLNLS1s favored the presence of relativistic jets, based on their high brightness temperatures, and their inverted radio spectra 
(Doi et al. 2007). Besides confirming the presence of relativistic jets, the previous work based on VLBA data argued that RLNLS1s can be either intrinsically radio loud, or apparently radio loud due to jet beaming effects ( $\mathrm{Gu} \& \mathrm{Chen}$ 2010; Doi et al. 2011). However, due to the very limited numbers of RLNLS1s observed with VLBI (e.g. Gu \& Chen 2010; Doi et al. 2011; Giroletti et al. 2011; D'Ammando et al. 2013; Wajima et al. 2014; Richards \& Lister 2015), it is far from clear that these features are universal in RLNLS1s.

Despite the rapidly growing number of RLNLS1s (Zhou et al. 2006), the mechanisms driving their radio properties are still unclear, including the role of accretion rate, black hole spin, circum-nuclear matter, host galaxy and merger history. In addition to radio observations, the detection of flaring $\gamma$-ray emission has confirmed the presence of relativistic jets in a few RLNLS1s (Abdo et al. 2009a, b). These observations may possibly be contrary to the well-known paradigm that jets are generally associated with elliptical host galaxies in typical radio-loud AGNs, while there is tentative evidence suggesting that at least a few lowredshift RLNLS1s are hosted by spiral galaxies (e.g. Zhou et al. 2007). Further, RLNLS1s generally have small black hole masses (e.g. Yao et al. 2015a), high accretion rates, and very strong Fe II emission (e.g. Komossa et al. 2006; Yuan et al. 2008), and are therefore at an opposite end of AGN correlation space than classical radio-loud AGNs (e.g., Sulentic et al. 2008).

With their remarkable multi-wavelength properties and extreme location in AGN parameter space, RLNLS1s allow us to re-address some of the key questions regarding the physics of jet formation, for example, the physical conditions under which a jet can be launched. Understanding the extreme properties (e.g., steep and flat spectra) shown simultaneously in the same class of objects, would be of help in determining the physical conditions of the jets, and in the core regions of these galaxies. Understanding the differences from other types of AGNs may then give further important insights into jet formation (e.g., Komossa et al. 2015). However, so far, little is known about the jets in RLNLS1, primarily due to the lack of systematic observations of their jets with high angular resolution.

In this paper, we present the first systematic high resolution radio study of RLNLS1s from the VLBA observations of 14 sources. The sample is presented in Section 2. The observations and data reduction is shown in Section 3, and the results are given in Section 4. Section 5 is for the discussion, and the last section is dedicated to conclusions. Throughout this paper, we assume a cosmology with $H_{0}=71 \mathrm{~km} \mathrm{~s}^{-1} \mathrm{Mpc}^{-1}, \Omega_{\mathrm{M}}=0.27$, and $\Omega_{\Lambda}=0.73$ (Spergel et al. 2003). The spectral index $\alpha$ is defined as $f_{\nu} \propto \nu^{\alpha}$, in which $f_{\nu}$ is the flux density at frequency $\nu$. 


\section{Sample}

To systematically study the radio structure at high resolution, we firstly compiled a large sample of RLNLS1s with the radio loudness parameter $R=f_{\nu}(1.4 \mathrm{GHz}) / f_{\nu}(4400 \AA) \geq 10$, by combining various samples available so far. The bulk of our sample is composed of

the RLNLS1s of Komossa et al. (2006), Zhou et al. (2006), and Yuan et al. (2008), totaling 99 objects. Furthermore, 18 sources from the compilation of Foschini (2011) are added in. The final sample thus consists of 117 RLNLS1s with $R \geq 10$, making it the largest RLNLS1 sample so far. Among the sample, only eight sources were detected by Fermi LAT (Abdo et al. 2009a, b; D'Ammando et al. 2012, 2015; Foschini 2011). We searched in the literature and found that, at that time, only 10 sources had published VLBI observations, six with Fermi detection and four without. We excluded these ten sources from our VLBA target list, along with two in the southern hemisphere (RX J0134-4258 and PKS 0558-504). As a result, our final RLNLS1 sample without published VLBI observations consists of 105 sources, all of which have $1.4 \mathrm{GHz}$ flux densities measured from the Faint Images of the Radio Sky at Twenty Centimeters (FIRST) 1.4-GHz radio catalogue (Becker et al. 1995). This is the first of a series of papers to systematically study the jet properties of RLNLS1s, aiming at gaining insights into the jet formation in these sources.

\section{Observations and data reduction}

As the first step of our systematic studies on the whole sample, we have performed VLBA observations with a total observing time of 15 hours for 16 sources on October and November, 2013 (program ID: BG217), selected with $1.4 \mathrm{GHz}$ flux densities $\geq 10 \mathrm{mJy}$ from 105 objects without published VLBI observations at that time. The key scientific goal is to image the putative relativistic jets in these objects at high resolution. Results have been obtained for 14 sources (including one Fermi object) with sufficient data quality to make images. The source list is shown in Table 1, in which the FIRST 1.4 GHz, the NRAO VLA Sky Survey (NVSS) $1.4 \mathrm{GHz}$ (Condon et al. 1998), the $5 \mathrm{GHz}$ flux density from the Green Bank 6-cm (GB6) survey (when available) (Gregorv et al.|1996), and the conventional radio loudness $R$ are given. All these sources are from Yuan et al. (2008), except for SDSS J142114.05+282452.8 from Foschini (2011).

Observations were made with the VLBA at the $\mathrm{C}$ band ranging from 3.9 to $7.9 \mathrm{GHz}$. We took the observational mode RDBE/DDC to use the largest recording rate of 2 Gbps, corresponding to a recording bandwidth of $256 \mathrm{MHz}$ in each of the two polarizations, to achieve sufficiently high imaging sensitivity. Dual circular polarizations were recorded at two widely separated frequencies of about $5 \mathrm{GHz}(4.868 \mathrm{GHz}$ at $4.804-4.932 \mathrm{GHz})$ and 6.7 
$\mathrm{GHz}(6.668 \mathrm{GHz}$ at $6.608-6.736 \mathrm{GHz})$ so that the polarization and spectral information may be obtained simultaneously. Sixteen target sources were observed in three separate blocks on Oct. 25, Nov. 14, and Nov. 24, 2013. The observations were made in phase referencing mode for three objects with the lowest flux densities (i.e. SDSS J085001.17+462600.5, SDSS J113824.54+365327.1 and SDSS J163401.94+480940.2, see Table 1). The on-source observing time was about 50 minutes for these three sources, while it was about 30 minutes for the remaining sources. Post data processing was performed within the package AIPS in a standard way for polarimetric VLBI observations, with the phase-referencing method applied to the three weakest sources. Due to its extremely low linear polarized emission, OQ 208 was successfully used to obtain the instrumental polarization in all the observations. As for the absolute polarized positional angle calibration, the calibrator 3C 286 was used but only a small part of the total flux density is restored, and hence only the fractional polarization images are given in this paper. It should be noted that the scaling factors in amplitude calibration for the left circular polarization of several antennas are obviously incorrect, therefore, we used the model of OQ 208 obtained from the right-handed polarization to calibrate the amplitude of the left-handed polarization, which proves to be quite successful. Imaging and model-fitting were done with DIFMAP, and the final results are given in Table 2. The typical uncertainties in the flux density and size are about $5 \%$, which is estimated by using the approximation in Fomalont (1999).

\section{Results}

\subsection{Morphology and brightness temperature}

The VLBA 5 GHz images for each object are shown in Figs. 1-14. We find that seven sources show a compact core only, while the remaining seven objects have core-jet structure. When available, we also present the images from archival VLBA data for these sources. The radio core of each source is identified as a component with a flat spectrum of $\alpha>-0.5$ tentatively estimated with 5 and $6.7 \mathrm{GHz}$ flux density from our measurements, and using archival VLBA multi-band data for several objects as well (see Section 4.3). All sources are compact with all components being within $\sim 5$ mas $(\sim 24-38$ pc depending on source redshift), except for two sources (SDSS J144318.56+472556.7 and SDSS J154817.92+351128.0) with extended jet emission at a distance of $>20$ mas (see Section 4.3). Our results thus indicate that the majority of our sources is compact at VLBI mas scales.

From the high-resolution VLBA images, the brightness temperature of the radio core $T_{\mathrm{B}}$ in the rest frame can be estimated with (Ghisellini et al. 1993) 


$$
T_{\mathrm{B}}=\frac{S_{\nu} \lambda^{2}}{2 k \Omega_{\mathrm{s}}}=1.77 \times 10^{12}(1+z)\left(\frac{S_{\nu}}{\mathrm{Jy}}\right)\left(\frac{\nu}{\mathrm{GHz}}\right)^{-2}\left(\frac{\theta_{d}}{\mathrm{mas}}\right)^{-2}
$$

in which $z$ is source redshift, $S_{\nu}$ is the core flux density at frequency $\nu$, and $\theta_{\mathrm{d}}$ is the source angular diameter $\theta_{\mathrm{d}}=\sqrt{\mathrm{ab}}$ where $\mathrm{a}$ and $\mathrm{b}$ are the major and minor axis, respectively. This estimate actually represents a lower limit on the brightness temperature. The Doppler factor of the jet $\delta$ can be usually constrained by

$$
T_{\mathrm{B}}^{\prime}=T_{\mathrm{B}} / \delta
$$

in which $T_{\mathrm{B}}^{\prime}$ is the intrinsic brightness temperature. Normally, $T_{\mathrm{B}}^{\prime}$ can be taken as the equipartition brightness temperature of $5 \times 10^{10} \mathrm{~K}$ (Readhead 1994), or the inverse Compton catastrophe brightness temperature of $\sim 10^{12} \mathrm{~K}$ (Kellermann \& Pauliny-Toth 1969). We note in passing that the Doppler factor estimated from radio observations is generally smaller than that from the SED modelling, specifically for $\gamma$-ray sources (e.g., Abdo et al. 2009b). This could be due to the fact that $\gamma$-rays are produced in very compact regions. In contrast, the radio emission is likely from much larger regions, in which the jet blob is expanded to be optically thin, therefore, it likely has decelerated (e.g., Blandford \& Königl 1979).

The core brightness temperature ranges from $10^{8.4}$ to $10^{11.4} \mathrm{~K}$ with a median value of $10^{10.1} \mathrm{~K}$ (see Table 2). This indicates that the radio emission is from non-thermal jets, confirming that powerful jets can be formed in accretion systems with relatively small black hole masses and high accretion rates (see e.g. Gu \& Chen 2010; Doi et al. 2011). In contrast, the VLBA core brightness temperatures of blazars typically range between $10^{11}$ and $10^{13} \mathrm{~K}$ with a median value near $10^{12} \mathrm{~K}$, and can even extend up to $5 \times 10^{13} \mathrm{~K}$ (Kovalev et al. 2005, 2009). While these measurements represent lower limits, the brightness temperatures of most our sources are below the equipartition and inverse Compton catastrophic limits. Therefore, the beaming effect is generally not significant in our sources. In principle, the strong Doppler beaming requires not only a nearly pole-on view but also a relativistic bulk jet speed. Thus, the lack of a strong beaming effect implies that the bulk jet speed may likely be low in our sources, which still needs to be confirmed with higher-resolution observations, since measured brightness temperatures are lower limits. Alternatively, it is possible that our sources are seen at slightly larger viewing angles. We find that the RLNLS1s with core only morphology generally have lower core and total flux density, lower redshift and lower brightness temperature than those of core-jet objects (see Fig. 15). This implies that the former likely have less powerful jets and are less beamed compared to the latter. 


\subsection{Polarization}

The fractional polarization images are given in Fig. 16 for five objects. We find that the fractional polarization in the jets $(\sim 10-30 \%)$ is evidently higher than that of the radio cores $(<5 \%)$ (see Fig. 16). This may plausibly be caused by the strong Faraday rotation in the nuclear region due to the high plasma density, and/or depolarization due to the complex core structure. The polarization is clearly asymmetric and there are fractional polarization variations in some regions of the jet. This is likely caused by the relatively strong jet-ISM interaction at the locations of high fractional polarization due to the jet bulk motion to that side, in the cases of SDSS J081432.11+560956.6, SDSS J090227.16+044309.6, SDSS J130522.75+511640.3, and SDSS J154817.92+351128.0. In SDSS J144318.56+472556.7, the polarization shows a prominent gradient along the jet direction, which well matches the jet knots. In this case, the surface shock may play an important role in the emission of these regions.

\subsection{Individual Objects}

Besides the VLBI radio structure, the radio spectrum is also important to study the source nature. The individual objects were investigated in detail by collecting the multifrequency and multi-epoch radio data from the NASA/IPAC Extragalactic Database (NED), in combination with the analysis on our VLBA images and archival VLBA data (if available). All sources were conventionally classified into flat-spectrum $(\alpha>-0.5$, in seven sources) and steep-spectrum ( $\alpha \leq-0.5$, in seven sources) RLNLS1s (see Table 1), by using the estimated radio spectral index $\alpha$ from low-resolution NED data (i.e. non-VLBI data), unless otherwise stated (see e.g., Sections 4.3.4 and 4.3.5).

\subsubsection{SDSS J081432.11+560956.6}

The source has a flat radio spectrum with a spectral index of $\alpha=-0.12$ based on $325 \mathrm{MHz}, 1.4$, and $4.85 \mathrm{GHz}$ data collected from NED. It could therefore be a blazar-like object. Our $5 \mathrm{GHz}$ image shows a core-jet structure with a weak jet component. The core is tentatively identified as the brightest component with an inverted spectrum of $\alpha=0.40$ between 5 and $6.7 \mathrm{GHz}$ from our data. The simultaneous VLBA images at 2.3 and $8.4 \mathrm{GHz}$ are presented in Fig. 1 by using the available visibility datal (Petrov 2013). The jet not

\footnotetext{
${ }^{1}$ http://astrogeo.org/obrs/obrs2_cat.html
} 
visually apparent at $2.3 \mathrm{GHz}$ is well resolved at $8.4 \mathrm{GHz}$. The core is confidently recognized as the brightest component with an inverted spectrum of $\alpha=0.18$ from the simultaneous 2.3 and $8.4 \mathrm{GHz}$ data (see Table 3), confirming the core identification at $5 \mathrm{GHz}$. In Fig. 1, we also show the VLBA $5 \mathrm{GHz}$ image from the archive, which was observed on May 31, 2006. A similar core-jet structure is seen. The core exhibits strong flux variability at $5 \mathrm{GHz}$ when comparing with our observations, from which the variability brightness temperature can be calculated (e.g. Yuan et al. 2008) to be $10^{10.3} \mathrm{~K}$, indicating no strong beaming effect, consistent with the low brightness temperature $10^{10.1} \mathrm{~K}$ from our $5 \mathrm{GHz}$ image (see Table 2). However, we emphasize that the core brightness temperatures of $10^{11.8}$ and $10^{11.5} \mathrm{~K}$ at 2.3 and $8.4 \mathrm{GHz}$ (see Table 3) are much higher, and therefore put much tighter constraints on the true brightness temperature. They do not yet exceed the inverse Compton catastrophe brightness temperature, though.

\subsection{2. $S D S S J 085001.17+462600.5$}

The source was detected in the Giant Metrewave Radio Telescope LBDS-Lynx Region 150-MHz Radio Source Catalog (Ishwara-Chandra et al. 2010) and with the Westerbork Northern Sky Survey (WENSS) at $325 \mathrm{MHz}$ (Rengelink et al. 1997), and it is compact in the FIRST image. The overall radio spectrum from $150 \mathrm{MHz}$ to $1.4 \mathrm{GHz}$ is steep with $\alpha=-0.62$. Therefore, the source is CSS-like. The spectrum is steep with a spectral index of -0.73 between $325 \mathrm{MHz}$ and $1.4 \mathrm{GHz}$. However it changes to flat with $\alpha=-0.34$, between 150 and $325 \mathrm{MHz}$, implying a turnover. The object has only a compact component in our VLBA image (see Fig. 2), which can be tentatively treated as core with an inverted spectrum between 5 and $6.7 \mathrm{GHz}$. We measure a brightness temperature of $10^{9.8} \mathrm{~K}$.

\subsubsection{SDSS J090227.16+044309.6}

The spectrum between $1.4 \mathrm{GHz}$ and $5 \mathrm{GHz}$ is flat with $\alpha=-0.24$ based on NED data. The source is resolved into core-jet structure, with the jet pointing towards the north-east at a position angle of about $60^{\circ}$ (see Fig. 3). The core brightness temperature from the VLBA image is $10^{11.4} \mathrm{~K}$. The total VLBA flux density at $5 \mathrm{GHz}(\sim 102 \mathrm{mJy})$ is very close to the GB6 flux (106 mJy), however only about half of that is from the radio core $(\sim 50 \mathrm{mJy})$. 


\subsubsection{SDSS J095317.09+283601.5}

The source is slightly resolved into a core-jet structure with a weak jet at position angle of about $33^{\circ}$ (see Fig. 4). The core brightness temperature is $10^{9.6} \mathrm{~K}$. The source was only detected at $1.4 \mathrm{GHz}$, and not detected in the GB6 survey. Taking the detection limit of GB6, $18 \mathrm{mJy}$, as the upper limit, the spectral index between 1.4 and $5 \mathrm{GHz}$ is expected to be steeper than -0.7 , implying a CSS-like nature, which is tentatively supported by the steep spectrum between $1.4 \mathrm{GHz}$ and VLBA $5 \mathrm{GHz}$ (total flux) with $\alpha=-0.93$. However, it is hard to draw firm conclusion on the source nature with present data, and further observations are needed to study the object in detail.

\subsubsection{SDSS J103727.45+003635.6}

The source was only detected at $1.4 \mathrm{GHz}$, so it is not possible to determine a radio spectral index with archival data. Combining the $1.4 \mathrm{GHz}$ measurement with our new, nonsimultaneous VLBA $5 \mathrm{GHz}$ flux density, yields a spectral index of about $\alpha \sim-0.20$. This source then could be a flat spectrum object. The VLBA flux is about $22 \mathrm{mJy}$, therefore, it could have been detected in the GB6 survey. The non-detection according to the GB6 catalog, then indicates that the source underwent variability, consistent with the flat radio spectrum. Only a compact component is detected in our VLBA image (Fig. 5), with brightness temperature of $10^{10.5} \mathrm{~K}$.

\subsubsection{SDSS J104732.68+472532.1}

The source is unresolved in the FIRST image, with upper limits on its extent of 1.16 and 1.14 arcsec for the major and minor axis, respectively, corresponding to a linear size of $\sim 8.7 \mathrm{kpc}$. The radio spectrum shows a clear trend for a turnover at low frequencies (see Fig. 17), with a spectral index of $\alpha=-0.12$ between 74 and $151 \mathrm{MHz}$, while it is steep $(\alpha=-0.53)$ at frequencies above $1.4 \mathrm{GHz}$. Such break frequencies at several hundred $\mathrm{MHz}$ in the source rest frame are often seen in small-scale radio sources, such as in CSSs (O'Dea 1998). This object thus resembles CSS sources in terms of the compact size and steep spectrum. We found the object is unresolved in our VLBA image, however a core-jet structure is obtained from archival VLBA $5 \mathrm{GHz}$ data because of higher resolution at eastwest direction (see Fig. 6). Intriguingly, the GB6 $5 \mathrm{GHz}$ flux density is about a factor of 20 higher than the total flux density of our VLBA image. The flux difference can either be

due to source variability, however this is unexpected in CSSs (O'Dea 1998), or else, we miss 
a significant fraction of the extended flux. For further investigations, we analysed the VLA images at 8.4 and $22.4 \mathrm{GHz}$ (see Fig. 6). A structure with two-sided lobes and a central core is clearly seen at a resolution of about 0.2 arcsec at $8.4 \mathrm{GHz}$, with a flux density ratio of about 1:1 between two lobes and the core (see Table 3). This implies that the source might be at large viewing angle, consistent with the steep spectrum. In contrast, only an unresolved compact component is present at $22.4 \mathrm{GHz}$ likely due to the poor resolution, much lower sensitivity, and/or steep spectrum of the extended jet emission. But still, compared with the expected VLA core flux density at $5 \mathrm{GHz}$ even assuming a spectral index of 0.0 between 5 and $8.4 \mathrm{GHz}$, our $5 \mathrm{GHz}$ flux density are still much smaller. If the flux density difference is not due to strong variability, then a high fraction of the flux density needs to be resolved out, possibly distributed in extended lobes in sizes between sub-arcsec and mas. The core brightness temperatures are $10^{8.4} \mathrm{~K}$, and $10^{9.0} \mathrm{~K}$ for our, and archival $5 \mathrm{GHz}$ images, respectively, consistent with a large viewing angle.

\subsubsection{SDSS J111005.03+365336.3}

Only a compact component is detected in our VLBA image (see Fig. 7), with brightness temperature of $10^{10.0} \mathrm{~K}$. The spectral index between WENSS $325 \mathrm{MHz}$ and $1.4 \mathrm{GHz}$ is steep, with $\alpha=-0.72$, making the source CSS-like. The spectrum remains steep, with $\alpha=-0.67$ between 1.4 and VLBA $5 \mathrm{GHz}$.

\subsubsection{SDSS J113824.54+365327.1}

Only a compact component is detected in our VLBA image (see Fig. 8), with a brightness temperature of $10^{9.5} \mathrm{~K}$. The spectral index between WENSS $325 \mathrm{MHz}$ and $1.4 \mathrm{GHz}$ is steep with $\alpha=-0.53$.

\subsubsection{SDSS J124634.65+023809.0}

Only a compact component is detected in our VLBA image (Fig. 9), with brightness temperature of $10^{9.7} \mathrm{~K}$. The spectral index between FIRST/NVSS $1.4 \mathrm{GHz}$ and GB6 $5 \mathrm{GHz}$ is inverted $(\alpha=0.17)$, making the source blazar-like. The VLBA flux $(\sim 9 \mathrm{mJy})$ is much less than that of the GB6 catalog (46 mJy). This flux difference can be either due to flux variations, or else most of flux is resolved out at VLBA resolution. 


\subsubsection{SDSS J130522.75+511640.3}

The spectral index based on low resolution radio data is steep with $\alpha=-0.56$ from 151 $\mathrm{MHz}$ to $4.85 \mathrm{GHz}$ (see Fig. 17). In combination with the compact FIRST structure (1.34 arcsec, i.e. $10 \mathrm{kpc}$ ), this indicates that this source is CSS-like. The source is resolved into two components in our $5 \mathrm{GHz}$ image (see Fig. 10). The northern component is brighter than the southern with a flux ratio of 1.7:1. The structure resembles that of compact symmetric objects, e.g. OQ 208 (Wu et al. 2013). However, the spectral index between 5 and $6.7 \mathrm{GHz}$ is flat $(\alpha=-0.41)$ for the northern component, while steep $(\alpha=-1.39)$ for the southern. This implies a core-jet structure for this source, rather than two steep-spectrum mini-lobes as in OQ 208. The core-jet structure is also supported by the inverted core spectrum of $\alpha \sim$ 0.29 between simultaneous VLBA 2.3 and $8.4 \mathrm{GHz}$, by analysing the available visibility data (Petrov 2013). The source is more resolved at 2.3 and $8.4 \mathrm{GHz}$ due to the higher resolution in north-south direction (see Fig. 10). The total VLBA $5 \mathrm{GHz}$ flux density ( $23.9 \mathrm{mJy})$ is only about half of that measured with GB6 (46 mJy), implying the other half is in the size between VLBA mas and GB6 resolution ( 3.5 arcmin) if the flux difference is not due to variability. The core brightness temperature is about $5.8 \times 10^{10} \mathrm{~K}$ at $5 \mathrm{GHz}$. In contrast, the brightness temperature is about $10^{11.2} \mathrm{~K}$ at both 2.3 and $8.4 \mathrm{GHz}$.

\subsubsection{SDSS J142114.05+282452.8}

The spectrum between FIRST/NVSS $1.4 \mathrm{GHz}$ and GB6 $5 \mathrm{GHz}$ is flat with $\alpha=-0.20$. The source is slightly resolved into a core-jet structure with a weak jet component. The simultaneous VLBA images at 2.3 and $8.4 \mathrm{GHz}$ are presented in Fig. 11 by using the available visibility data (Petrov 2013). The weak jet is also seen in the $2.3 \mathrm{GHz}$ image, however, it is not present in the $8.4 \mathrm{GHz}$ image. The core is identified as the brightest 2.3 $\mathrm{GHz}$ component with a flat spectrum of $\alpha=-0.33$ between 2.3 and $8.4 \mathrm{GHz}$. The core brightness temperature is about $9.5 \times 10^{10} \mathrm{~K}$ at $5 \mathrm{GHz}$, and $10^{10.6}$ and $10^{10.1} \mathrm{~K}$ at 2.3 and $8.4 \mathrm{GHz}$, respectively.

\subsubsection{SDSS J144318.56+472556.7}

The multi-frequency NED data show that the spectral index between $151 \mathrm{MHz}$ and 4.85 $\mathrm{GHz}$ is steep with $\alpha=-0.60$ (see Fig. 17). The source is not listed in the Very Large Array

Low-frequency Sky Survey Redux catalog (VLSSr, Lane et al. 2014), which only contains the sources with $74 \mathrm{MHz}$ flux density at greater than $5 \sigma$ significance, i.e. $\sim 0.5 \mathrm{Jy}_{\text {beam }}{ }^{-1}$. 
Taking $0.5 \mathrm{Jy}$ as the upper limit at $74 \mathrm{MHz}$, the radio spectrum shows a clear trend of a turnover toward low frequencies (Fig. 17). Indeed, the spectral index is -0.57 between 151 and $408 \mathrm{MHz}$, while it is -0.77 between 1.4 and $4.85 \mathrm{GHz}$. This object is unresolved in the FIRST image with a size of 1.18 arcsec (major axis), corresponding to a linear size of 8.4 kpc. Given its steep spectrum and compact structure, this NLS1 shares similarity with CSS sources. The VLBA $5 \mathrm{GHz}$ image is shown in Fig. 12. The source is resolved into seven components along the south-west direction within 15 mas ( $107 \mathrm{pc})$ (see Table 2), and diffuse emission is clearly detected at large distance up to 30 mas. Tentatively calculating the spectral index between 5 and $6.7 \mathrm{GHz}$ from our VLBA data, we found that the spectra of all seven components are steep. This implies that the radio core is not directly observed, perhaps hidden in the brightest component. However, multi-frequency VLBI observations are needed before we can draw firm conclusions. The brightness temperature at $5 \mathrm{GHz}$ is $10^{10.3} \mathrm{~K}$ for the brightest component. The total flux density at $5 \mathrm{GHz}$ from our VLBA image is close to that in the single dish GB6 catalog (see Fig. 17), which indicates that the source is very compact, and there is likely no much extended emission even at sub-arcsec scale. The extended and/or diffuse emission can be only resolved at mas scale, which contains a large fraction of the jet emission as the flux density of the brightest component is only about $\sim 40 \%$ of the total flux density.

\subsubsection{SDSS J154817.92+351128.0}

The source is well resolved into a core-jet structure (see Fig. 13). The jet is along the south-north direction in the central region, then it turns to the north-west. At a large distance of about 60 mas $(\sim 353 \mathrm{pc})$, diffuse emission is evident. The core is identified with the brightest component, with an inverted spectral index of $\alpha=0.4$, tentatively estimated from our 5 and $6.7 \mathrm{GHz}$ measurements. However, its $5 \mathrm{GHz}$ flux density is only about $42 \%$ of the total flux density. The source was detected at $74 \mathrm{MHz}$ in the VLSSr catalog. The multi-wavelength data shows an overall flat spectrum $(\alpha=-0.43)$ from $74 \mathrm{MHz}$ all the way to $8.4 \mathrm{GHz}$, and the spectrum is flat $(\alpha=-0.45)$ above $1.4 \mathrm{GHz}$ (see Fig. 17). The overall radio spectrum shows that this source is most likely a flat-spectrum source, resembling blazars. However, there perhaps is a turnover below $100 \mathrm{MHz}$, possibly due to synchrotron self-absorption. The total VLBA flux density (33 mJy) is about $55 \%$ of that of VLA (60 $\mathrm{mJy}$, from NED), indicating that about half of the jet emission is resolved out at VLBA resolution. However, alternatively, the flux difference between VLBA and VLA can also be due to the flux variations since it is a flat-spectrum object. The brightness temperature of the core is $10^{9.9} \mathrm{~K}$ at $5 \mathrm{GHz}$. A variability brightness temperature of $10^{13} \mathrm{~K}$ was found at $4.85 \mathrm{GHz}$ by Yuan et al. (2008), which implies a Doppler factor of $\delta_{\min }=2.2$. 


\subsubsection{SDSS J163401.94+480940.2}

Only a compact component is detected in our VLBA image (see Fig. 14), with brightness temperature of $10^{10.1} \mathrm{~K}$. The spectrum between WENSS $325 \mathrm{MHz}$ and NVSS $1.4 \mathrm{GHz}$ data of similar resolution is flat with $\alpha=-0.47$. The FIRST flux density is only about $55 \%$ of NVSS, implying that the remaining flux is either resolved out at FIRST resolution, or else the source is variable.

\section{Discussion}

All our sources were selected with $1.4 \mathrm{GHz}$ flux densities $\geq 10 \mathrm{mJy}$, and they are very radio loud with $R>100$ (see Table 1 ). Therefore, they represent the most extreme RLNLS1s in terms of their radio loudness $R$. However, their jet properties are not homogeneous, in terms of the mas scale morphology and the overall radio spectral shape. A variety of mas scale morphologies has been found, including compact core only (7 out of 14), compact core-jet (5 of 14), and extended core-jet structures (2 of 14). Based on the compactness defined as the core to total flux ratio, most sources (12 of 14) are compact, and coredominated with compactness larger than 0.5. Two sources, SDSSJ144318.56+472556.7 and SDSSJ154817.92+351128.0, have a compactness of $\sim 0.4$ with a large-scale jet extending to about 30 and 60 mas (see Figs. 12 and 13), respectively. A one-sided core-jet structure is detected in all the resolved objects (7 out of 14), which is commonly found in blazars. As for the radio spectral shape, we found flat spectra in seven sources, and steep spectra in the other seven sources, albeit large uncertainties in SDSS J095317.09+283601.5 and SDSS J103727.45+003635.6 (see Section 4.3 and Table 1). In our sample, there is no strong relationship between the compactness and spectral index. The steep spectrum sources do not prefer to have more extended structures, while those with flat spectra do not appear more compact.

It should be noted that our measurements of brightness temperature are typically lower limits, and the true brightness temperatures can therefore be higher. However, taken at face value, the median brightness temperature of our sample is significantly lower than that of typical blazars (of order $10^{12} \mathrm{~K}$; Kovalev et al. 2005, 2009). If confirmed by future higherresolution observations, this finding can, in principle, be explained in two ways. Either, our sources are not significantly beamed, then likely implying a low bulk speed of the jet. This is likely the case for flat-spectrum sources, since the viewing angles are expected to be small in these sources. Alternatively, these sources are seen at relatively large angle, and Doppler-deboosting plays a role, which could be the situation in steep-spectrum objects (e.g., SDSS J104732.68+472532.1, see Section 4.3.6). Indeed, although not conclusive due 
to the lower limit, the brightness temperatures of steep spectrum sources are systematically lower than those of flat spectrum sources with median values of $10^{9.8}$ and $10^{10.1} \mathrm{~K}$ for steep and flat sources (see Fig. 15), respectively. This is qualitatively consistent with the expectation that flat spectrum sources are usually viewed at relatively smaller angles, and then have a relatively larger beaming effect than steep spectrum sources. Moreover, the steep-spectrum RLNLS1s were recently proposed as the parent population of flat-spectrum RLNLS1s (Berton et al. 2015a). In this scenario, the fundamental jet physics could be similar, such as the jet bulk speed, and the observational differences in two populations could be mainly due to the different viewing angle. Therefore, given the overall properties of our sources, the first possibility is favored that the jet bulk speed is likely low in our sources. Consistently, the radio monitoring of $\gamma$-ray-detected NLS1s shown that the computed variability brightness temperatures are in general moderate, in contrast to the majority of blazars, implying mildly relativistic jets (Angelakis et al. 2015). The mildly relativistic jets were also found in Richards \& Lister (2015) for three RLNLS1s based on various information, such as the moderate core brightness temperature, the jet/counter-jet intensity ratio and the extension length ratios from VLBA and VLA observations.

Flux variability of each source was searched for by comparing the FIRST and NVSS fluxes. Due to the different beam sizes of NVSS and FIRST, we conservatively treat the sources with the FIRST peak flux larger than the integrated flux of NVSS as possible variable sources. The significance of the variation $\sigma_{\mathrm{var}}$ is calculated as the ratio of the flux difference between the FIRST peak flux and NVSS integrated flux to the combined uncertainties including the corresponding uncertainties in FIRST and NVSS fluxes and the additional systematic uncertainties (see e.g. Wang et al. 2006). Using a threshold $\sigma_{\text {var }}>3$, we found flux variations in only one source, SDSSJ085001.17+462600.5 $\left(\sigma_{\mathrm{var}}=4.0\right)$. For most sources (10/14), the FIRST peak and integrated fluxes are in agreement with the integrated NVSS flux within $10 \%$. The mean values of the flux ratio of FIRST to NVSS are $0.99 \pm 0.17$ and $1.03 \pm 0.19$, for FIRST peak and integrated fluxes, respectively. This again shows that variations are usually not significant for most sources. Moreover, it indicates that all our sources are mostly compact, without much extended diffuse emission, as often found in RLNLS1s (Doi et al. 2012; Yuan et al. 2008). Indeed, all fourteen NLS1 galaxies of our sample were detected as single unresolved sources within 1 arcmin of the SDSS position in both FIRST and NVSS, indicating compact radio structure at FIRST resolution ( 5 arcsec), except if any extended emission was faint, and beyond the detection threshold of NVSS and FIRST. We tentatively use the deconvolved major axis provided in the FIRST catalog as an upper limit of the linear size of each source. The linear sizes range from 2.1 to $17.4 \mathrm{kpc}$ (see Table 1), confirming that they are compact.

The association of RLNLS1s with CSS sources has already been proposed by many 
authors (Moran et al. 2000; Oshlack et al. 2001; Gallo et al. 2006; Komossa et al. 2006), and recently by Caccianiga et al. (2014). In addition, the hypothesis of CSS sources as the parent population of RLNLS1s was also discussed, in which the RLNLS1s with a flat radio spectrum could be CSS sources with jets pointing towards the observer (Yuan et al. 2008; Caccianiga et al. 2014). Indeed, the steep-spectrum RLNLS1s were recently found to likely be the best candidates for the parent population of flat-spectrum RLNLS1s, although not conclusive and disk-hosted radio galaxies have yet to be included (Berton et al. 2015a). In our sample, seven sources have compact structure and steep spectrum (although uncertain in the case of SDSS J095317.09+283601.5, see Section 4.3). These share some resemblance with CSS sources. However, their VLBI structure is different from that of typical CSS sources (O'Dea 1998; Dallacasa et al. 2013). As shown in Dallacasa et al. (2013), the majority of CSS sources have a two-sided structure and the radio emission is dominated by lobes, jets and hotspots. In our sample, four out of seven steep spectrum sources have core-only morphology, and a one sided core-jet structure is found in the remaining three objects. For core-jet sources, the core flux dominates in two objects ( 90\% in SDSSJ095317.09+283601.5, 63\% in SDSSJ130522.75+511640.3). Only in SDSSJ144318.56+472556.7, the radio emission is dominated by the elongated jet structure and diffuse emission with about $40 \%$ of the total emission in the core. While most of the high-power typical CSS sources have a double or triple morphology at VLBI scales (O’Dea 1998; Dallacasa et al. 2013), compact core only and core-jet structures have been detected in CSS sources with relatively low radio power (Kunert-Bajraszewska et al. 2006; Kunert-Bajraszewska \& Marecki 2007). In terms of the linear size (using FIRST major axis as upper limit) and $5 \mathrm{GHz}$ radio power (Yuan et al. 2008), our NLS1 galaxies resemble the low-power CSS sources of Kunert-Bajraszewska et al. (2010). In the radio power versus linear size diagram, the low-power CSS objects occupy the space below the main evolutionary path of radio objects. The authors argued that many of these might be short-lived objects, and their radio emission may be disrupted several times before they become FR IIs. Alternatively, some of the short-lived low-power CSS objects could be precursors to large-scale FR Is (see also An \& Baan 2012). The argument linking RLNLS1s with CSS sources mainly relies on the compact arcsec scale structure in RLNLS1s. The majority of RLNLS1s exhibit compact radio emission, while extended emission has only been detected in nine RLNLS1s (Doi et al. 2012; Richards \& Lister 2015). Doi et al. (2012) argued that the jet of RLNLS1s has low kinetic power because of the small mass and because it has to propagate in a gas-rich environment, which can be likely responsible for rare detection of extended emission. However, recent VLA observations of three arbitrarily selected RLNLS1s, show that all sources possess kpc-scale extended emission (Richards \& Lister 2015). One of the CSS-like sources in our sample, SDSS J095317.09+283601.5, was reported to have a two-sided, mildly core-dominated jet structure with diffuse lobes at an overall projected linear size of about $70.2 \mathrm{kpc}$ in their study, largely exceeding the typical size of CSS sources. 
The authors argued that the extended emission could be more common when observed with enhanced sensitivity, such as the upgraded VLA. While this indicates the capability of jet extension to kpc scale, more observations are needed to study the fraction of extended emission in the overall RLNLS1 population.

With NVSS, polarized flux was detected in 11 out of the 14 NLS1 galaxies of our sample. The fractional polarization ranges from $0.5 \%$ to $5.2 \%$ with a mean value of $2.2 \%$. There is no significant systematic difference of fractional polarization between steep and flat spectrum sources. In the steep spectrum sources, the fractional polarization is similar to that of CSS sources ( $\sim 1 \%-3 \%$ at $6 \mathrm{~cm}$, see O'Dea 1998). Several studies of the polarization properties of CSS sources have shown that sources smaller than a few kpc have very low values of polarized emission at frequencies below $8.4 \mathrm{GHz}$ (e.g. Cotton et al. 2003). This result has been interpreted by assuming that compact sources are highly depolarized by the dense interstellar medium that enshrouds the radio emission and acts as a Faraday screen. When using the deconvolved major axis as the upper limit of the source linear size, we found that the fractional polarization of steep spectrum sources is below $2 \%$ for objects smaller than $10 \mathrm{kpc}$, while higher than $4 \%$ for larger sources. This suggests that there may be an abrupt change in the properties of the ISM at a radius of approximately $10 \mathrm{kpc}$, possibly related to the outer boundary of the narrow line region (NLR), which however needs further studies. The radio power distribution of our RLNLS1 galaxies overlaps with the lower part of that of CSSs (O'Dea 1998). As such, if there is an overlap between CSSs and RLNLS1 galaxies, it should consist mostly of CSSs having low radio power and relatively small black hole masses.

Besides seven steep spectrum sources in our sample, seven galaxies show flat radio spectra, implying relationships with blazars. Recently, Foschini et al. (2015) conducted a multi-wavelength survey of 42 RLNLS1s with flat radio spectra. They found that the jet power is generally lower than FSRQs and BL Lac objects, but partially overlapping with the latter, consistent with the results of Sun et al. (2015). In fact, once normalised by the mass of the central black holes, the jet powers are consistent with each other for these three types of AGNs, indicating the scalability of the jet. The authors argued that the central engine of RLNLS1s is apparently quite similar to that of blazars, despite the observational differences. Based on the detailed analysis of the $\gamma$-ray flux variability and spectral properties of five RLNLS1s, Paliva et al. (2015) found that these $\gamma$-NLS1s are similar to FSRQs in terms of their average $\gamma$-ray photon index, however their $\gamma$-ray luminosities are lower than powerful FSRQs but higher than BL Lac objects. In view of the curvature in the $\gamma$-ray spectrum and flux variability amplitudes, the authors suggested that these $\gamma$-ray NLS1s could be similar to powerful FSRQs, but with low or moderate jet power. Although similarities have been found between RLNLS1s and blazars, we cannot yet perform a comparison between the host galaxies of blazars (commonly found in ellipticals) and the RLNLS1s of our sample. All 
our sources are at relatively high redshift, and their SDSS images are insufficient for host analysis.

It has been suggested that outflows in AGNs can be accelerated by the radiation pressure of the accretion disk (e.g., Murray et al. 1995), which could be important for the jet production in sources accreting at rates near the Eddington limit, as commonly found in NLS1 galaxies (e.g., Grupe et al. 2010; Xu et al. 2012). However, Cao (2014) found that it is difficult for the outflows to be directly driven from the photospheres of the radiation-dominated disks, which implies that hot gas (probably in the corona) is necessary for launching an outflow from the radiation-pressure-dominated disk. The authors investigated outflows accelerated from the hot corona above the disk by the magnetic field and radiation force of the accretion disk, and found that with the help of the radiation force, the mass-loss rate in the outflow is high, which however leads to a slow outflow. In our RLNLS1s sources, the accretion rate is high, and the radiation pressure could be significant. Therefore, the scenario of Cao (2014) might be relevant in explaining why the jets in RLNLS1s are in general mildly relativistic compared with those in blazars. Recently, Berton et al. (2015b) proposed that the jet in RLNLS1s can be possibly launched by a rapidly accreting disk along with the winds when the angle between the poloidal component of the magnetic field and the accretion disk is less than a critical angle.

Alternatively, in the Blandford-Znajek (BZ) mechanism, the jet energy and angular momentum can be extracted from a rotating black hole (Blandford \& Znajek 1977). The low jet speed in RLNLS1s is consistent with the significant correlation between black hole mass and the jet bulk Lorentz factor found in typical blazars by Chai et al. (2012), as the black hole masses of RLNLS1s are systematically lower than blazars. This correlation can be well explained in a black hole spin scenario, in the sense that the faster moving jets are magnetically accelerated by the magnetic fields threading the horizon of more rapidly rotating black holes (Chai et al. 2012). Recent investigation indeed suggested that most supermassive black holes in elliptical galaxies (e.g. for FSRQs) have on average higher spins than the black holes in spiral galaxies, where random, small accretion episodes (e.g., tidally disrupted stars, accretion of molecular clouds) might have played a more important role (Volonteri et al. 2007). If RLNLS1s do follow the correlation of Chai et al. (2012), the low jet speed in RLNLS1s may then be due to the low spin. Few actual BH spin estimates for NLS1 galaxies are available to date, and none for the radio-loudest NLS1 galaxies. Based on deep X-ray observations of a few nearby (radio-quiet) NLS1 galaxies, intermediate to very high spins were estimated (e.g., Miniutti et al. 2009; Fabian et al. 2012, 2013; Parker et al. 2014; Gallo et al. 2015), while Done et al. (2013) preferred a low-spin solution, based on SED fitting of the NLS1 galaxy PG 1244+026. Liu et al. (2015) reported indications for low or intermediate average BH spin, estimated from stacked X-ray spectra of a larger sample 
of 51 NLS1 galaxies. Estimates for radio-loud NLS1 galaxies are not yet available.

Up to now, less than ten RLNLS1s were detected in $\gamma$-rays with Fermi/LAT (see Abdo et al. 2009a, b; D'Ammando et al. 2012, 2015; Foschini 2011; Yao et al. 2015b). The only source in our sample with a tentative $\gamma$-ray detection with Fermi, SDSS J124634.65+023809.0, has the steepest spectrum with a $\gamma$-ray photon index of $\Gamma=3.1$, however with the lowest Test Statistic $(\mathrm{TS}=15)$ (Foschini 2011). This source shows compact core-only morphology (see Fig. 9), different from the common core-jet structure in other $\gamma$-ray sources (e.g. Orienti et al. 2012). The brightness temperature of $10^{9.7} \mathrm{~K}$ is significantly lower compared to other $\gamma$-ray sources, which is usually larger than $10^{11} \mathrm{~K}$ (e.g., PMN J0948+0022 in Giroletti et al. 2011). This is somewhat in contrast to the notion that the $\gamma$-ray objects tend to have larger beaming effects than non- $\gamma$-ray detected ones, for example, as suggested for BL Lac objects in Wu et al. (2014). Finally, we would like to point out that the majority of the $\gamma$-ray detected NLS1 galaxies have published VLBI observations, and were therefore not re-analyzed in the study presented here. Therefore, our sample likely excludes a few of the most variable, and most highly beamed sources. We will present the results for a sample of fainter sources in a forthcoming paper based on the VLBA observations ( $\mathrm{Gu}$ et al. 2015, in prep.). Moreover, in order to directly constrain the jet bulk speed, multi-epoch VLBA observations have been planned to detect the jet proper motion for a sample of RLNLS1s with resolved core-jet structure. All these observations will provide us with a deeper understanding on the jet formation in these low black hole mass, and high accretion rate systems.

\section{Conclusions}

As the first step of our systematic studies of the pc scale radio structure of RLNLS1s, we have obtained VLBA observations of 14 very radio-loud NLS1 galaxies in 2013. These observations reveal that the radio morphology of the sources is generally compact. Although all these NLS1 galaxies are very radio-loud objects with $R>100$, their jet properties are diverse, in terms of the mas scale morphology and overall radio spectral shape. The low core brightness temperatures, if confirmed with higher resolution, indicate that beaming effects are generally not significant, likely implying a low jet speed in these sources. This, in combination with the low kinetic/radio power, is likely responsible for the compact VLBA radio structure in most sources. The mildly relativistic jet in these high accretion rate systems could likely be explained by the scenario that jets/outflows are accelerated from the hot corona above the disk by the magnetic field and radiation force of the accretion disk, in which the jets/outflows are slow. Alternatively, the low jet bulk velocity can be explained by the Blandford-Znajek mechanism, if the black holes have low spin. 
We thank the anonymous referee for constructive comments that improved the manuscript. We thank Xinwu Cao, Tao An and Thomas Krichbaum for valuable discussions. This work is supported by the National Science Foundation of China (grants 11473054, 11273042, 11473035, 11173046, and U1531245) and by the Science and Technology Commission of Shanghai Municipality (grants 14ZR1447100 and 12ZR1436100), the Strategic Priority Research Program "The Emergence of Cosmological Structures" of the Chinese Academy of Sciences (Grant No. XDB09000000), and the Strategic Priority Research Program on Space Science, the Chinese Academy of Sciences (Grant No. XDA04060700). This study makes use of data from the SDSS (see http://www.sdss.org/collaboration/credits.html).

Facilities: VLBA

\section{REFERENCES}

Abdo A. A. et al., 2009a, ApJ, 699, 976

Abdo A. A. et al., 2009b, ApJ, 707, L142

An, T., \& Baan, W. A. 2012, ApJ, 760, 77

Angelakis, E., Fuhrmann, L., Marchili, N., et al. 2015, A\&A, 575, A55

Becker R. H., White R. L., Helfand D. J., 1995, ApJ, 450, 559

Berton, M., Foschini, L., Ciroi, S., et al. 2015a, A\&A, 578, A28

Berton, M., Foschini, L., Ciroi, S., et al. 2015b, arXiv:1506.05800

Blandford, R. D., \& Znajek, R. L. 1977, MNRAS, 179, 433

Blandford R. D., Königl A., 1979, ApJ, 232, 34

Caccianiga, A., Antón, S., Ballo, L., et al. 2014, MNRAS, 441, 172

Cao, X. 2014, ApJ, 783, 51

Chai, B., Cao, X., \& Gu, M. 2012, ApJ, 759, 114

Condon, J. J., Cotton, W. D., Greisen, E. W., et al. 1998, AJ, 115, 1693

Cotton, W. D., Dallacasa, D., Fanti, C., et al. 2003, PASA, 20, 12

Dallacasa, D., Orienti, M., Fanti, C., Fanti, R., \& Stanghellini, C. 2013, MNRAS, 433, 147 
D'Ammando, F., Orienti, M., Finke, J., et al. 2012, MNRAS, 426, 317

D'Ammando, F., Orienti, M., Doi, A., et al. 2013, MNRAS, 433, 952

D’Ammando, F., Orienti, M., Larsson, J., \& Giroletti, M. 2015, MNRAS, 452, 520

Doi A. et al., 2007, PASJ, 59, 703

Doi, A., Asada, K., \& Nagai, H. 2011, ApJ, 738, 126

Doi, A., Nagira, H., Kawakatu, N., et al. 2012, ApJ, 760, 41

Done, C., Jin, C., Middleton, M., \& Ward, M. 2013, MNRAS, 434, 1955

Fabian, A. C., Zoghbi, A., Wilkins, D., et al. 2012, MNRAS, 419, 116

Fabian, A. C., Kara, E., Walton, D. J., et al. 2013, MNRAS, 429, 2917

Fomalont, E. B. 1999, Synthesis Imaging in Radio Astronomy II, 180, 301

Foschini, L. 2011, Narrow-Line Seyfert 1 Galaxies and their Place in the Universe, 24

Foschini, L., Berton, M., Caccianiga, A., et al. 2015, A\&A, 575, A13

Gallo L. C. et al., 2006, MNRAS, 370, 245

Gallo, L. C., Wilkins, D. R., Bonson, K., et al. 2015, MNRAS, 446, 633

Ghisellini, G., Padovani, P., Celotti, A., \& Maraschi, L. 1993, ApJ, 407, 65

Giroletti, M., Paragi, Z., Bignall, H., et al. 2011, A\&A, 528, LL11

Goodrich R.W., 1989, ApJ, 342, 22

Gregory P. C., Scott W. K., Douglas K., Condon J. J., 1996, ApJS, 103, 427

Grupe, D., Komossa, S., Leighly, K. M., \& Page, K. L. 2010, ApJS, 187, 64

Gu, M., \& Chen, Y. 2010, AJ, 139, 2612

Ishwara-Chandra, C. H., Sirothia, S. K., Wadadekar, Y., Pal, S., \& Windhorst, R. 2010, MNRAS, 405, 436

Kellermann K. I., Pauliny-Toth I. I. K., 1969, ApJ, 155, L71

Komossa S., Voges W., Xu D., Mathur S., Adorf H. M., Lemson G., Duschl W., Grupe D., 2006, AJ, 132, 531 
Komossa S., 2008, Rev. Mex. AA Ser. Conf., 32, 86

Komossa, S., Xu, D., Fuhrmann, L., et al. 2015, A\&A, 574, A121

Kovalev, Y. Y., Kellermann, K. I., Lister, M. L., et al. 2005, AJ, 130, 2473

Kovalev, Y. Y., Aller, H. D., Aller, M. F., et al. 2009, ApJ, 696, L17

Kunert-Bajraszewska, M., Marecki, A., \& Thomasson, P. 2006, A\&A, 450, 945

Kunert-Bajraszewska, M., \& Marecki, A. 2007, A\&A, 469, 437

Kunert-Bajraszewska, M., Gawroński, M. P., Labiano, A., \& Siemiginowska, A. 2010, MNRAS, 408, 2261

Lane, W. M., Cotton, W. D., van Velzen, S., et al. 2014, MNRAS, 440, 327

Liu, Z., Yuan, W., Lu, Y., \& Zhou, X. 2015, MNRAS, 447, 517

Miniutti, G., Panessa, F., de Rosa, A., et al. 2009, MNRAS, 398, 255

Moran E. C., 2000, NewA Rev., 44, 527

Murray, N., Chiang, J., Grossman, S. A., \& Voit, G. M. 1995, ApJ, 451, 498

O’Dea, C. P. 1998, PASP, 110, 493

Orienti, M., D’Ammando, F., Giroletti, M., \& for the Fermi-LAT Collaboration 2012, arXiv:1205.0402

Oshlack, A. Y. K. N., Webster, R. L., \& Whiting, M. T. 2001, ApJ, 558, 578

Osterbrock D. E., Pogge R. W., 1985, ApJ, 297, 166

Paliya, V. S., Stalin, C. S., \& Ravikumar, C. D. 2015, AJ, 149, 41

Parker, M. L., Wilkins, D. R., Fabian, A. C., et al. 2014, MNRAS, 443, 1723

Petrov, L. 2013, AJ, 146, 5

Readhead A.C.S., 1994, ApJ, 426, 51

Rengelink, R. B., Tang, Y., de Bruyn, A. G., et al. 1997, A\&AS, 124, 259

Richards, J. L., Lister, M. L., Savolainen, T., et al. 2015, IAU Symposium, 313, 139

Richards, J. L., \& Lister, M. L. 2015, ApJ, 800, L8 
Spergel, D. N., Verde, L., Peiris, H. V., et al. 2003, ApJS, 148, 175

Sulentic, J. W., Zamfir, S., Marziani, P., \& Dultzin, D. 2008, Revista Mexicana de Astronomia y Astrofisica Conference Series, 32, 51

Sun, X.-N., Zhang, J., Lin, D.-B., et al. 2015, ApJ, 798, 43

Volonteri, M., Sikora, M., \& Lasota, J.-P. 2007, ApJ, 667, 704

Wajima, K., Fujisawa, K., Hayashida, M., et al. 2014, ApJ, 781, 75

Wang, T.-G., Zhou, H.-Y., Wang, J.-X., Lu, Y.-J., \& Lu, Y. 2006, ApJ, 645, 856

Wu, F., An, T., Baan, W. A., et al. 2013, A\&A, 550, A113

Wu, Z., Jiang, D., Gu, M., \& Chen, L. 2014, A\&A, 562, A64

Xu, D., Komossa, S., Zhou, H., et al. 2012, AJ, 143, 83

Yao, S., Yuan, W., Komossa, S., et al. 2015a, AJ, 150, 23

Yao S., Yuan W., Zhou H., et al. 2015b, MNRAS, in press

Yuan W., Zhou H. Y., Komossa S., Dong X. B., Wang T. G., Lu H. L., Bai J. M., 2008, ApJ, 685, 801

Zhou H., Wang T., Yuan W., Lu H., Dong X., Wang J., Lu Y., 2006, ApJS, 166, 128

Zhou H. et al., 2007, ApJ, 658, L13 

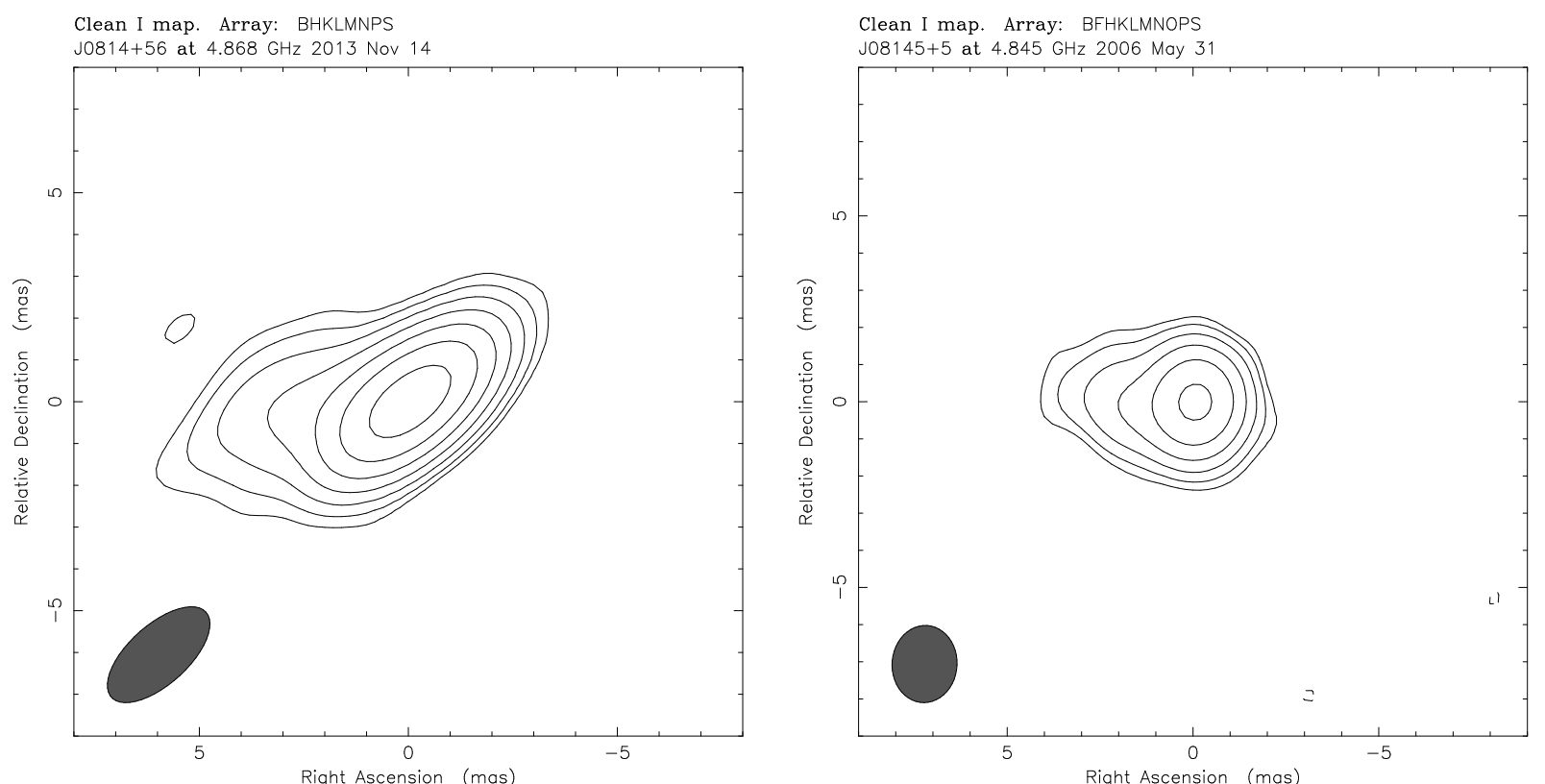

Map center: RA: 0814 32.135, Dec: +56 0956550 (2000.0)

Map peak: $0.0205 \mathrm{Jy} /$ beam

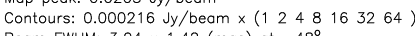

Beam FWHM: $3.04 \times 1.42$ (mas) at $-48^{\circ}$

Clean RR map. Array: VLBA Correlator

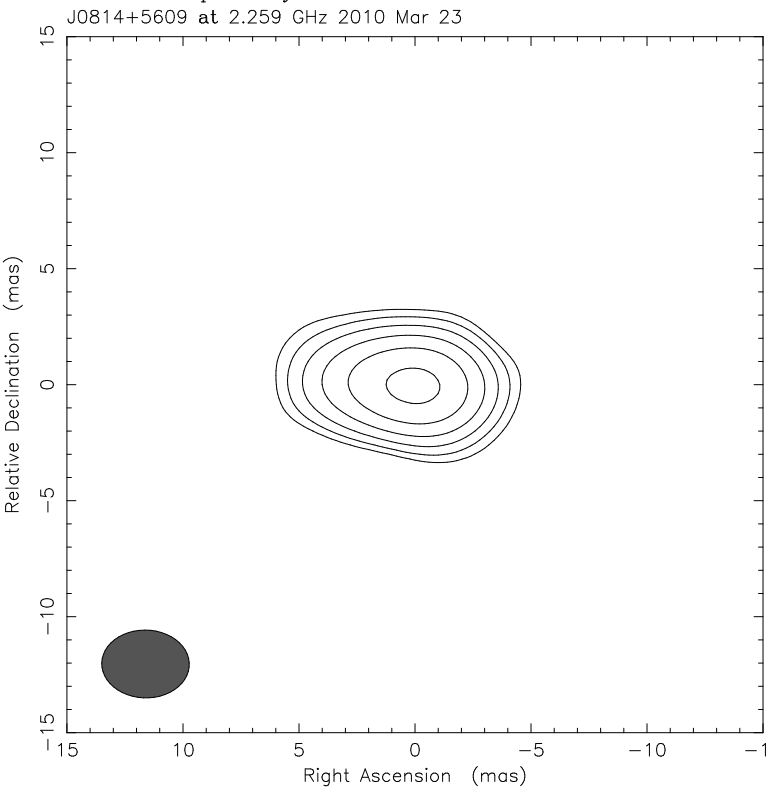

Map center: RA: 0814 32.116, Dec: +56 0956.687 (2000.0)

Map peak: $0.0254 \mathrm{Jy} /$ beam

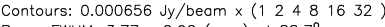

Beam FWHM: $3.77 \times 2.92$ (mas) at $88.7^{\circ}$

Map center: RA: 0814 32.115, Dec: +56 0956.681 (2000.0)

Map peak: $0.0292 \mathrm{Jy} /$ beam

Contours: 0.000784 Jy/beam x (-1 $\left.\begin{array}{llllll}-1 & 2 & 4 & 8 & 16 & 32\end{array}\right)$

. $2.08 \times 1.75$ (mas) at $-3.75^{\circ}$

Clean RR map. Array: VLBA Correlator J0814+5609 at $8.409 \mathrm{GHz} 2010$ Mar 23

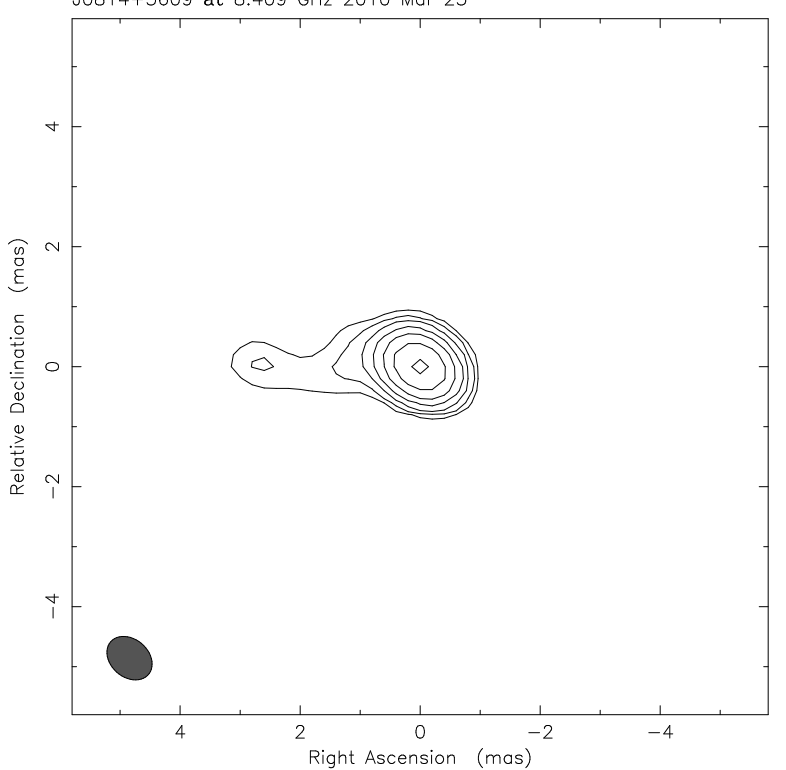

Map center: RA: 0814 32.116, Dec: +56 0956.687 (20000)

Map peak: $0.0308 \mathrm{Jy} /$ beam

Contours: $0.000425 \mathrm{Jy} /$ beam $\times\left(\begin{array}{llllll}1 & 2 & 4 & 8 & 16 & 32 \\ & 64\end{array}\right)$

Beam FWHM: $0.833 \times 0.632$ (mas) at $49.1^{\circ}$

Fig. 1. - VLBA $5 \mathrm{GHz}$ image of SDSS J081432.11+560956.6, and archival 2.3, 5 and 8.4 $\mathrm{GHz}$ VLBA images. 


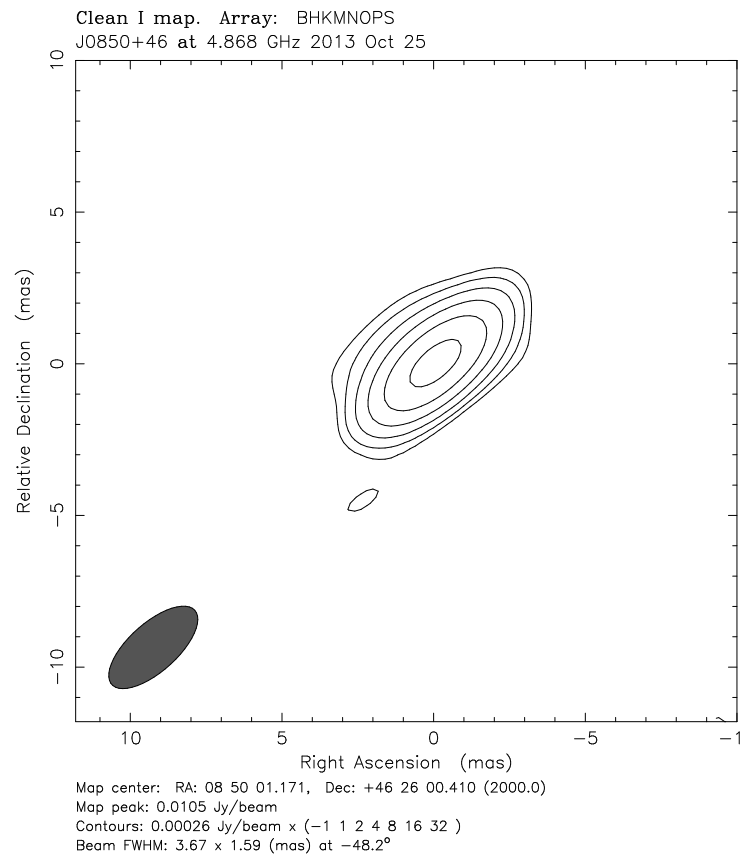

Fig. 2.- VLBA 5 GHz image of SDSS J085001.17+462600.5.

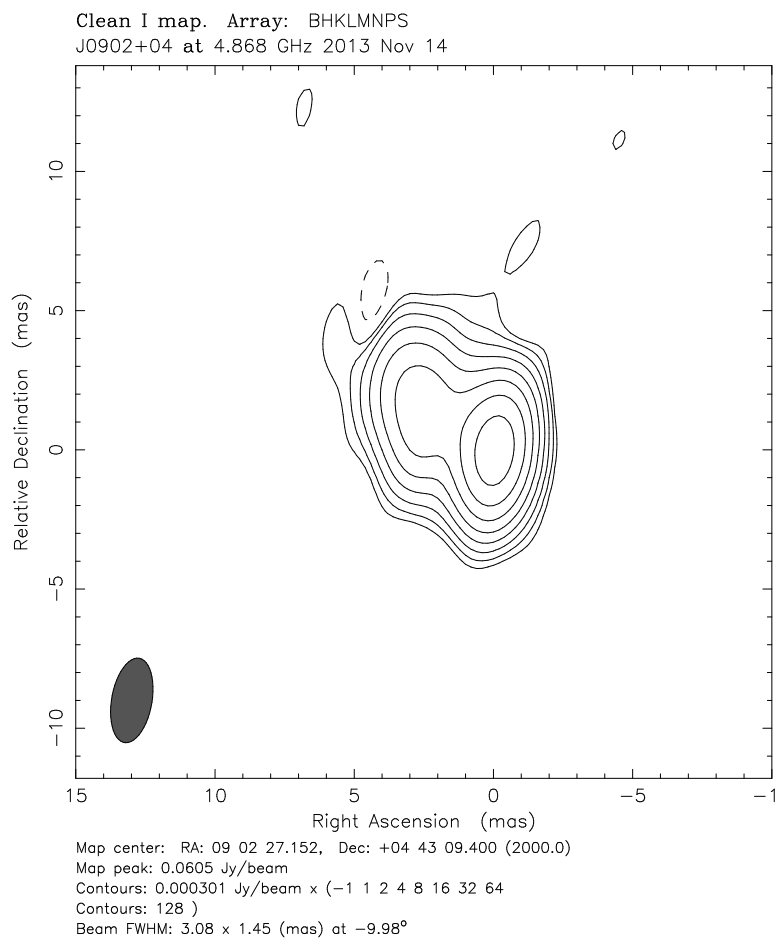

Fig. 3.- VLBA 5 GHz image of SDSS J090227.16+044309.6. 


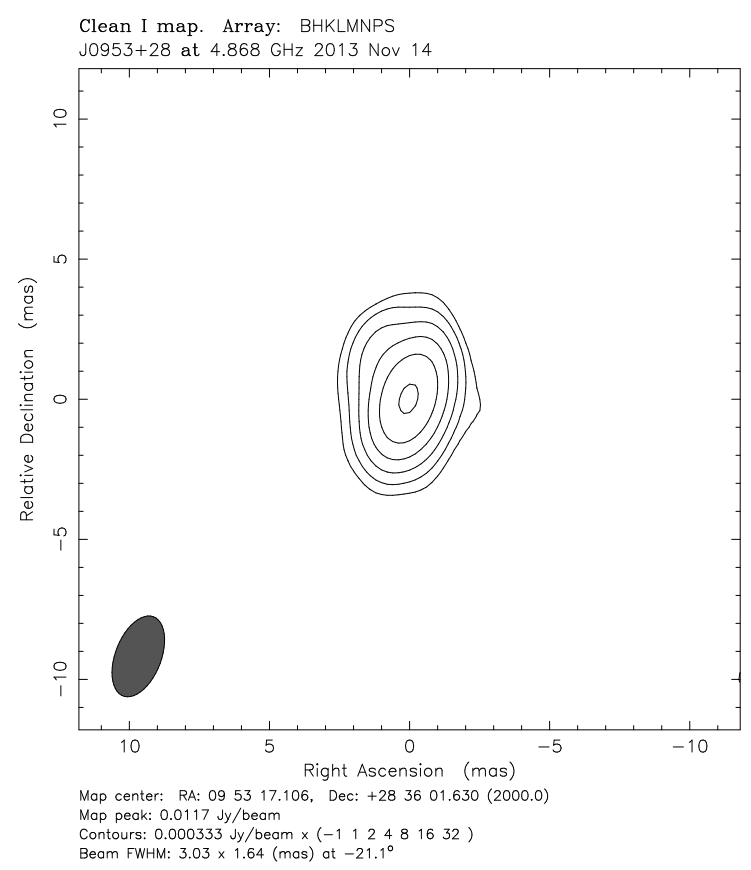

Fig. 4. - VLBA 5 GHz image of SDSS J095317.09+283601.5.

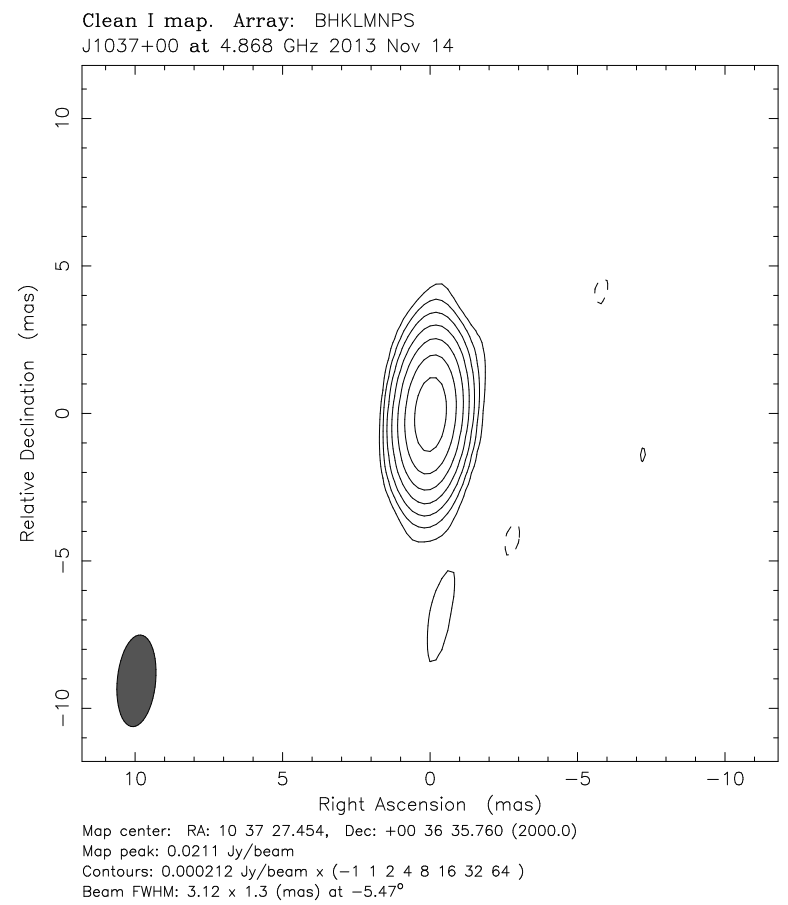

Fig. 5.- VLBA 5 GHz image of SDSS J103727.45+003635.6. 

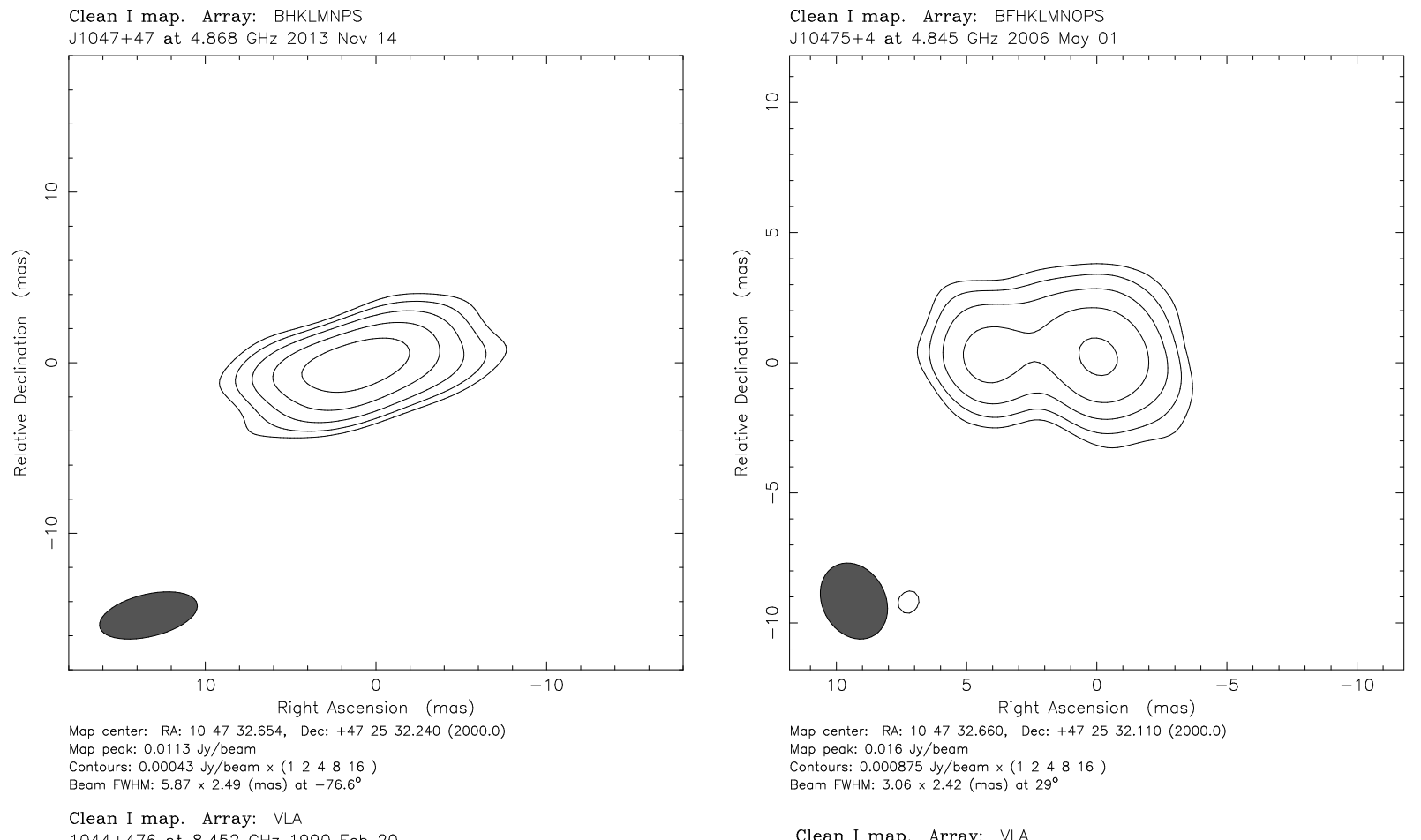

Map center: RA: 104732.660 , Dec: $+472532.110(2000.0)$ Map peak: $0.016 \mathrm{Jy} /$ bear

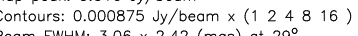

Clean I map Array: VLA

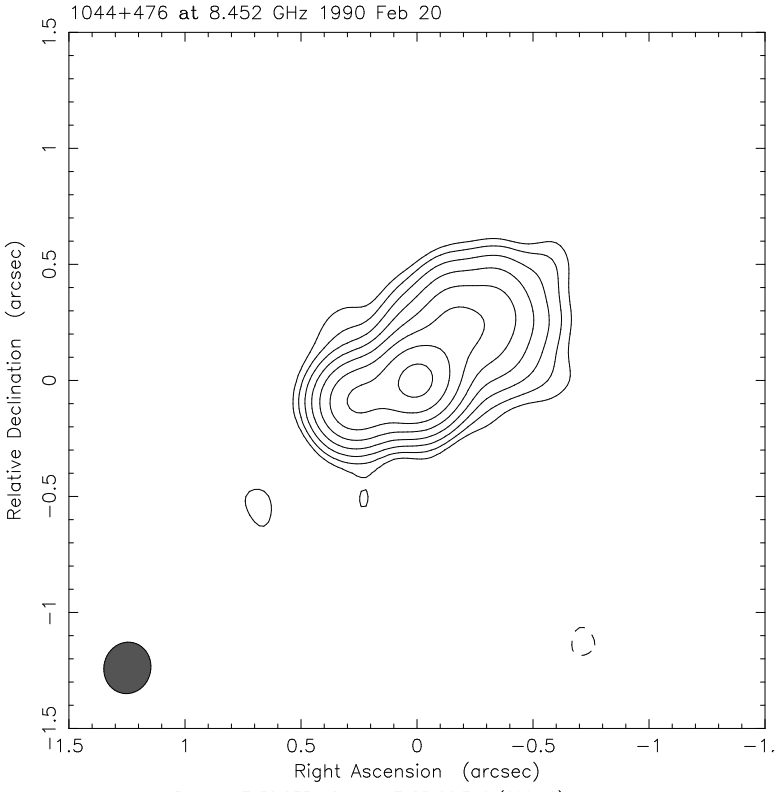

Map center: RA: 104732.833 , Dec: $+472522.798(2000.0)$

Map peak: $0.131 \mathrm{Jy} /$ beam

Contours: 0.000700 Jy/beam $\times(-112248163254$

Bean FWHM: $0.223 \times 0.202$ (arsec) at $-13^{0}$

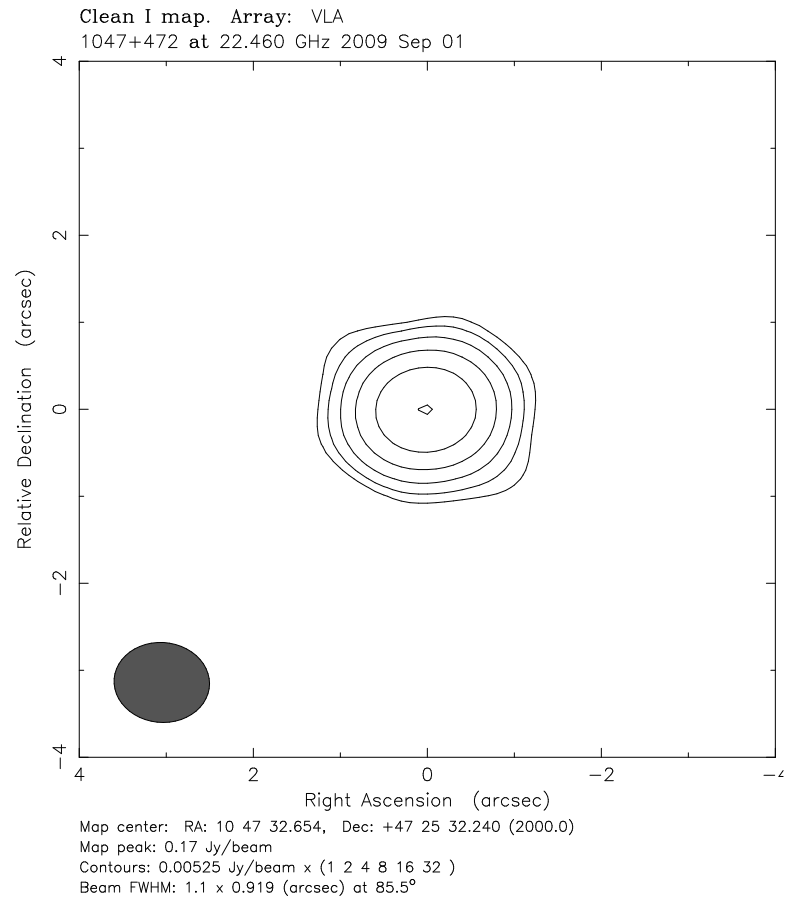

Fig. 6. - VLBA 5 GHz image of SDSS J104732.68+472532.1, archival VLBA 5 GHz image, and VLA images at 8.4 and $22.4 \mathrm{GHz}$. 


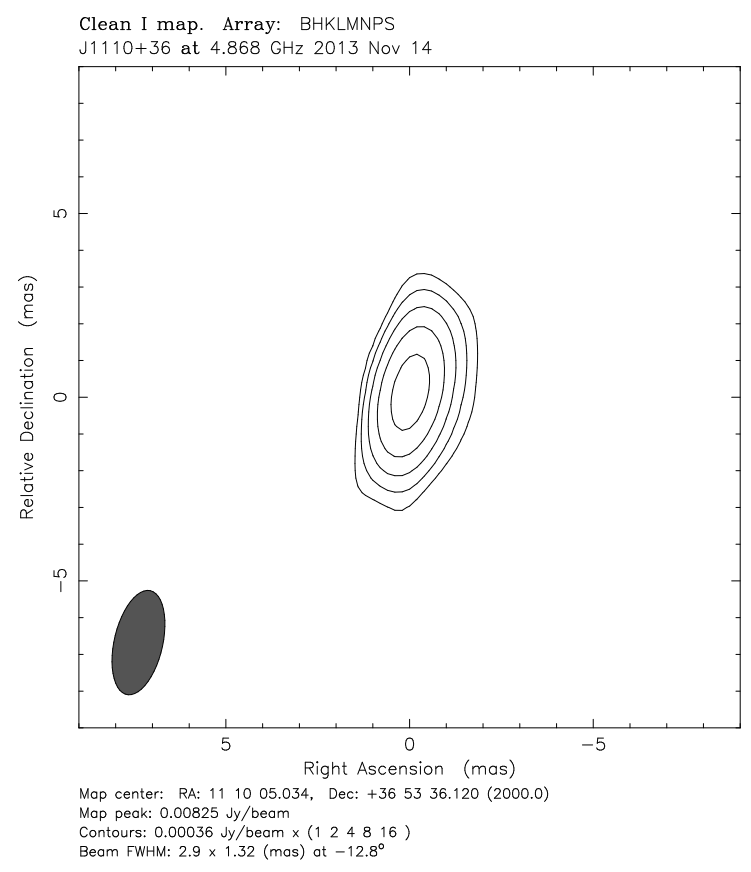

Fig. 7.- VLBA 5 GHz image of SDSS J111005.03+365336.3

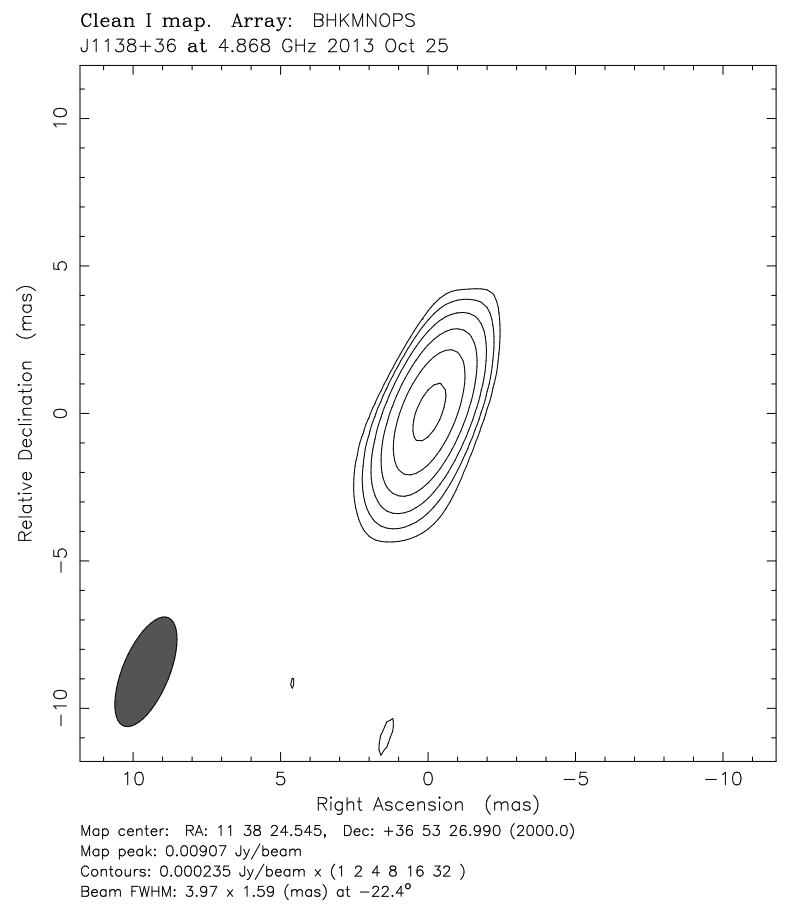

Fig. 8. - VLBA 5 GHz image of SDSS J113824.54+365327.1. 


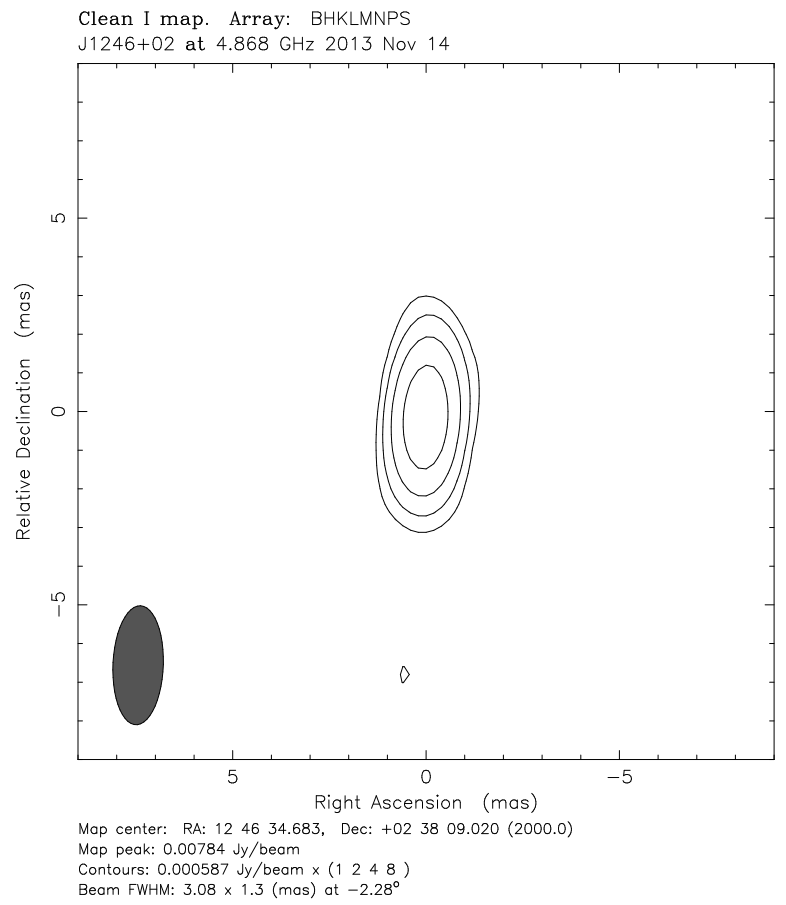

Fig. 9.- VLBA 5 GHz image of SDSS J124634.65+023809.0. 

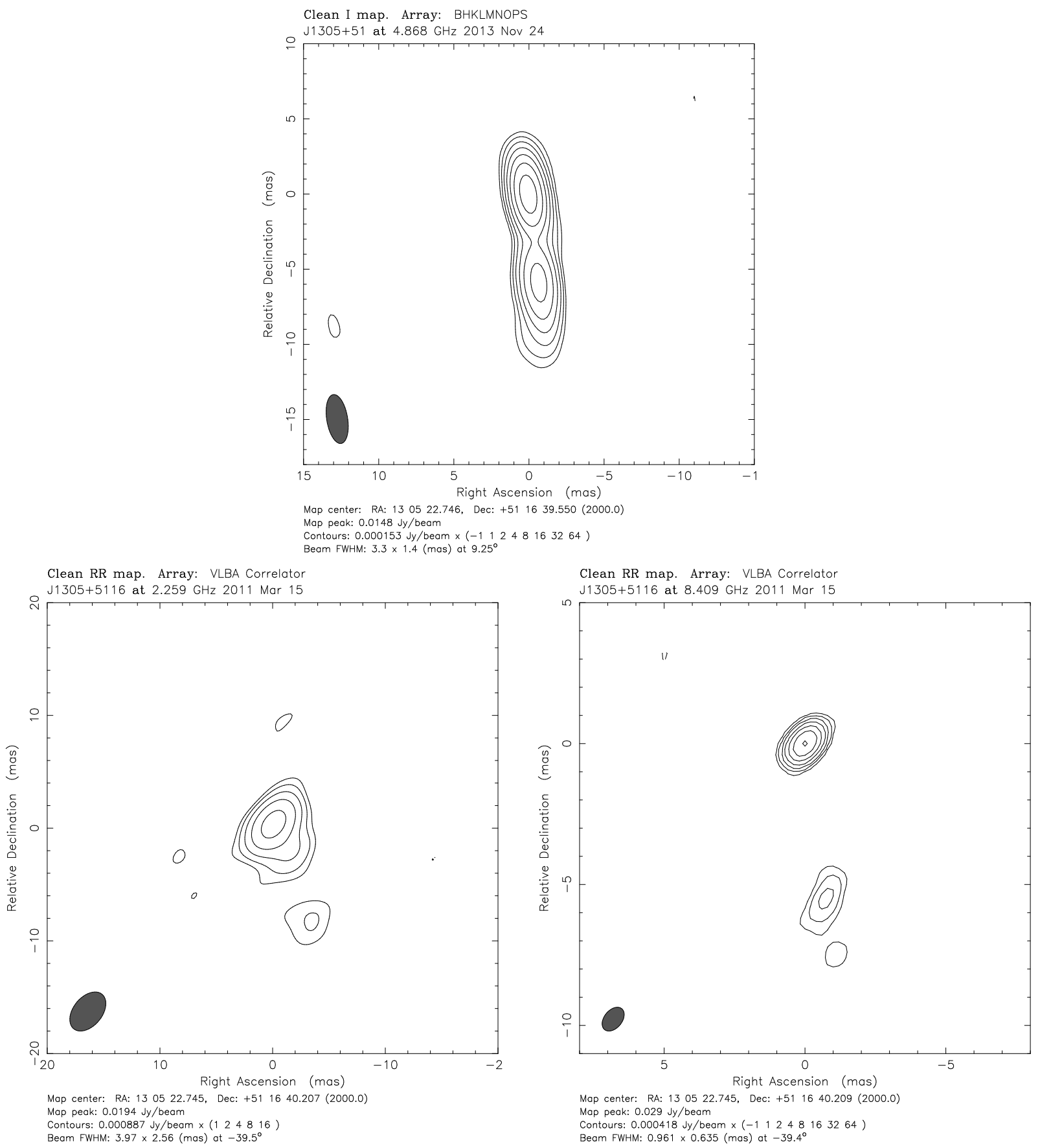

Fig. 10. - VLBA 5 GHz image of SDSS J130522.75+511640.3, and archival VLBA 2.3 and $8.4 \mathrm{GHz}$ images. 

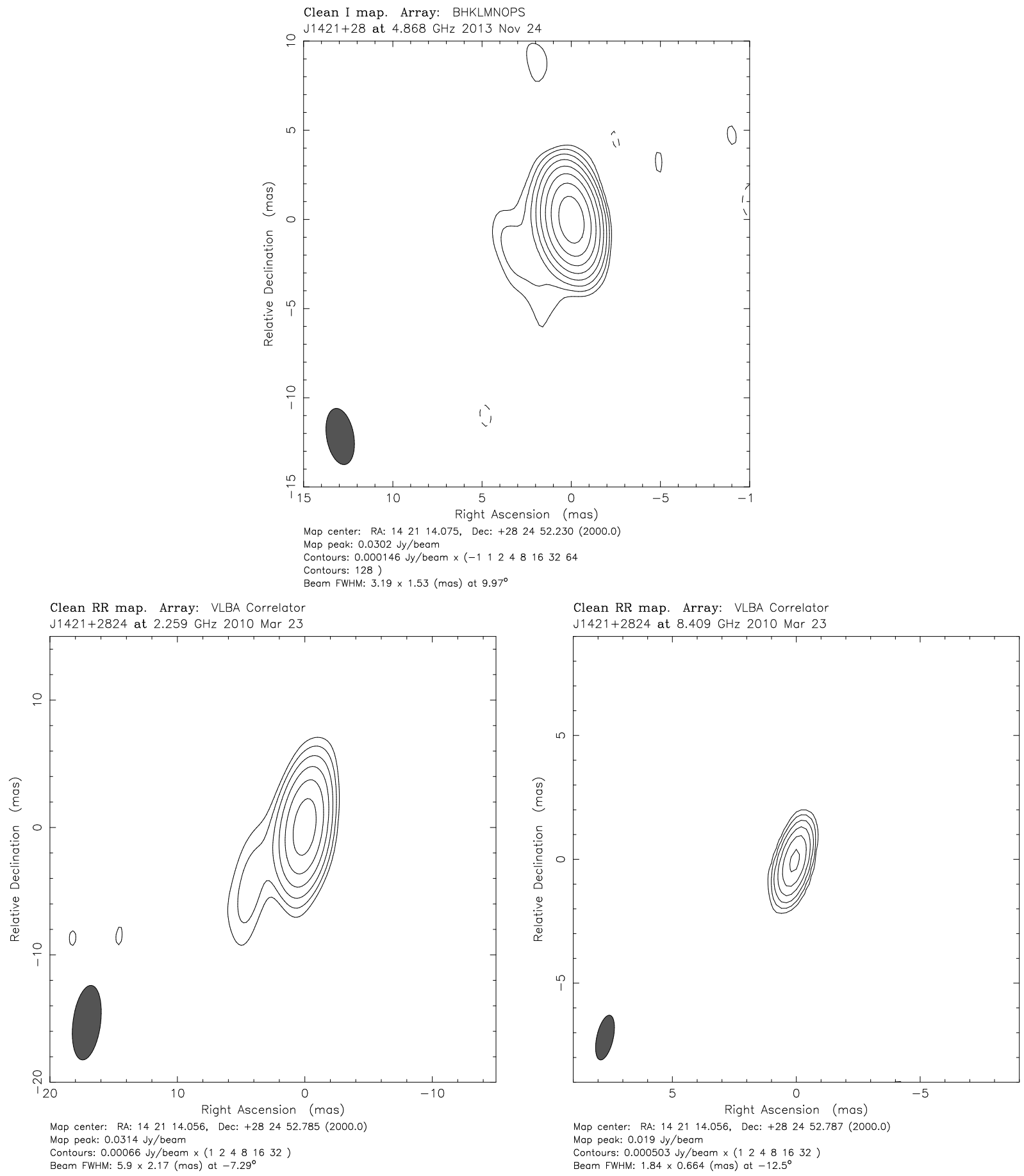

Fig. 11. - VLBA 5 GHz image of SDSS J142114.05+282452.8, and archival VLBA 2.3 and $8.4 \mathrm{GHz}$ images. 


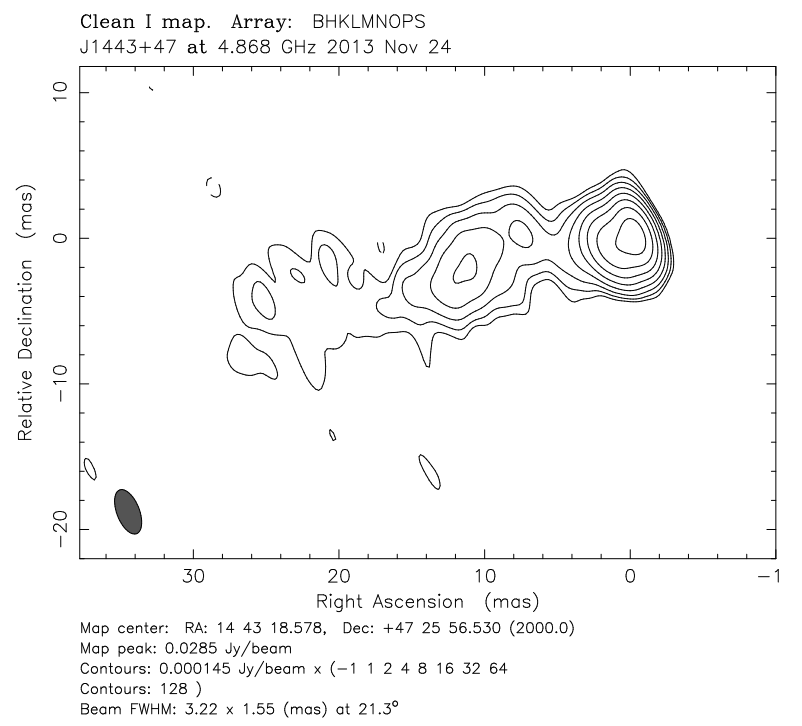

Fig. 12. - VLBA 5 GHz image of SDSS J144318.56+472556.7.

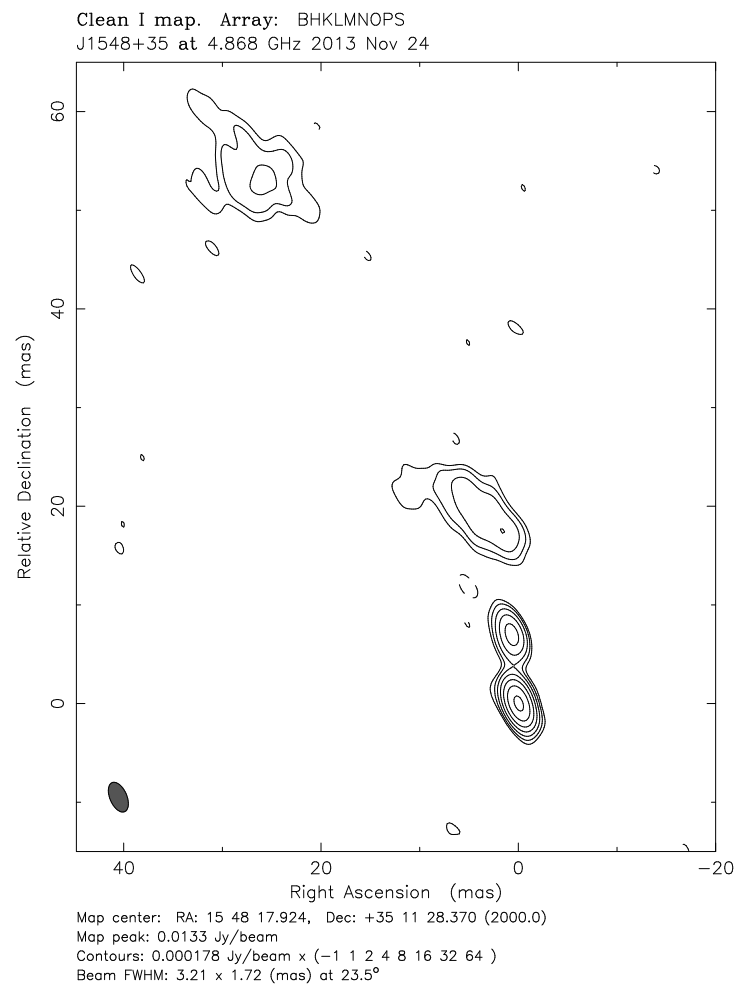

Fig. 13.- VLBA 5 GHz image of SDSS J154817.92+351128.0. 


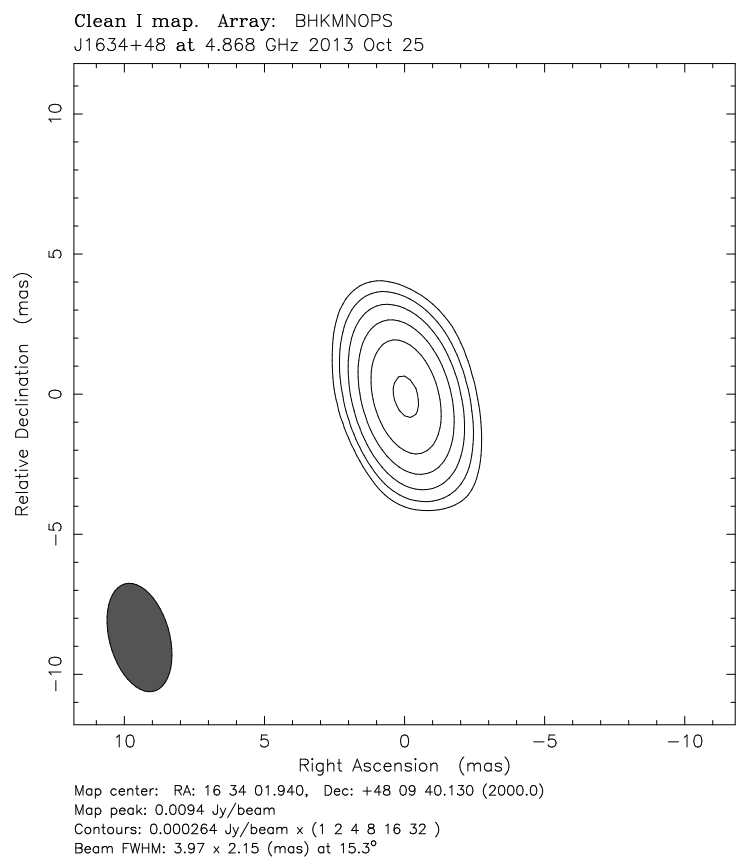

Fig. 14.- VLBA 5 GHz image of SDSS J163401.94+480940.2.
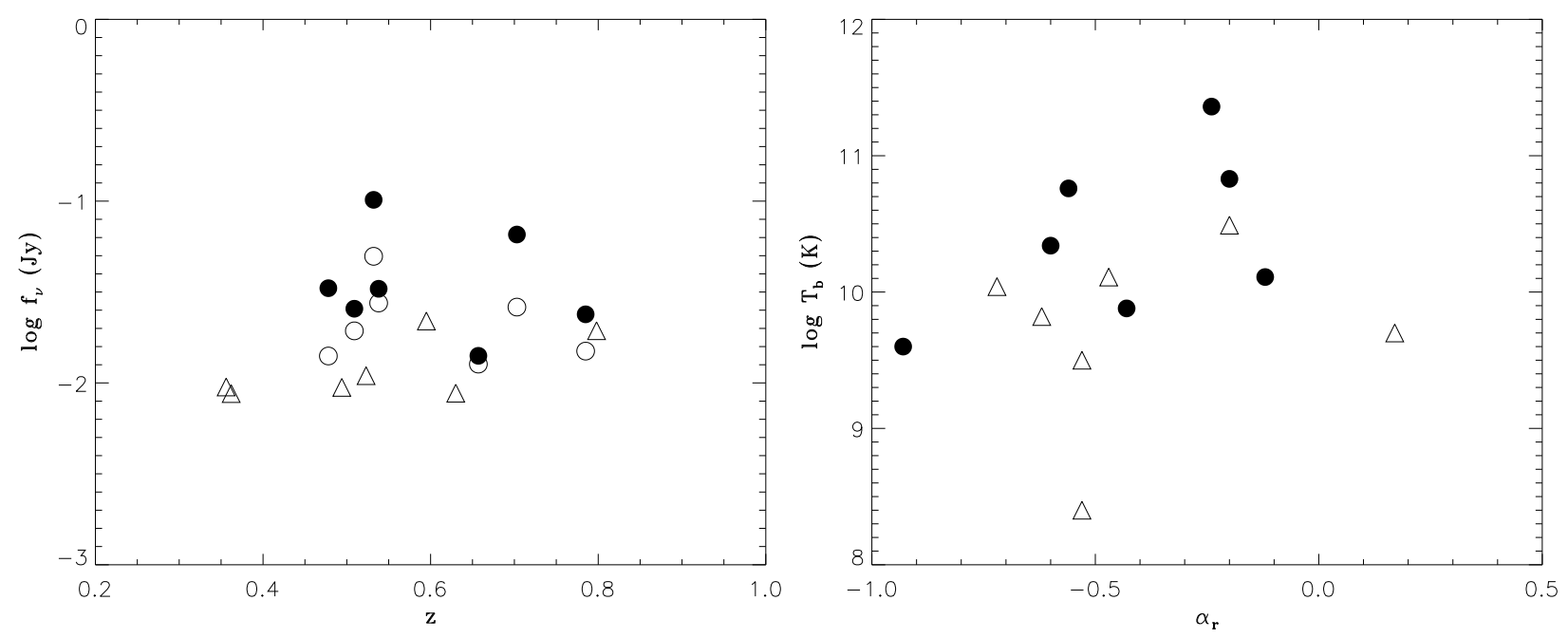

Fig. 15.- Left: Source redshift versus flux density. Triangles are for objects with core only (and then the flux densities are for the cores). The circles are sources with core-jet morphology. The solid circles represent the overall source flux density, while the open circles are for the core only. Right: Core brightness temperature versus radio spectral index based on low-resolution NED data (see text for details). The circles are for core-jet sources, while the triangles represent core-only objects. 

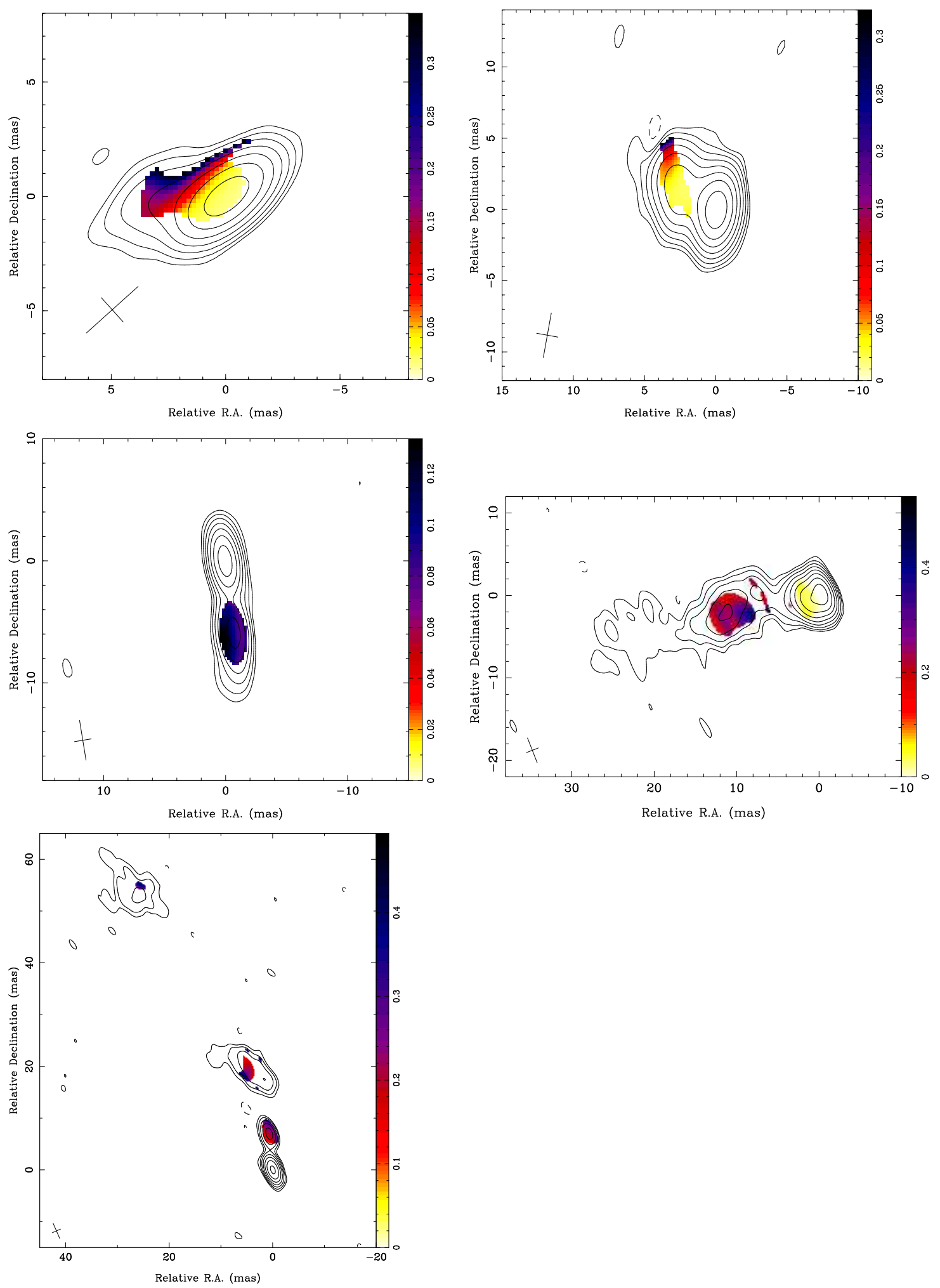

Fig. 16. - Fractional polarization. Upper left - SDSS J081432.11+560956.6; upper right SDSS J090227.16+044309.6; middle left - SDSS J130522.75+511640.3; middle right - SDSS J144318.56+472556.7; lower left - SDSS J154817.92+351128.0. In each panel, the contour represents the overall VLBA radio structure in total intensity. 

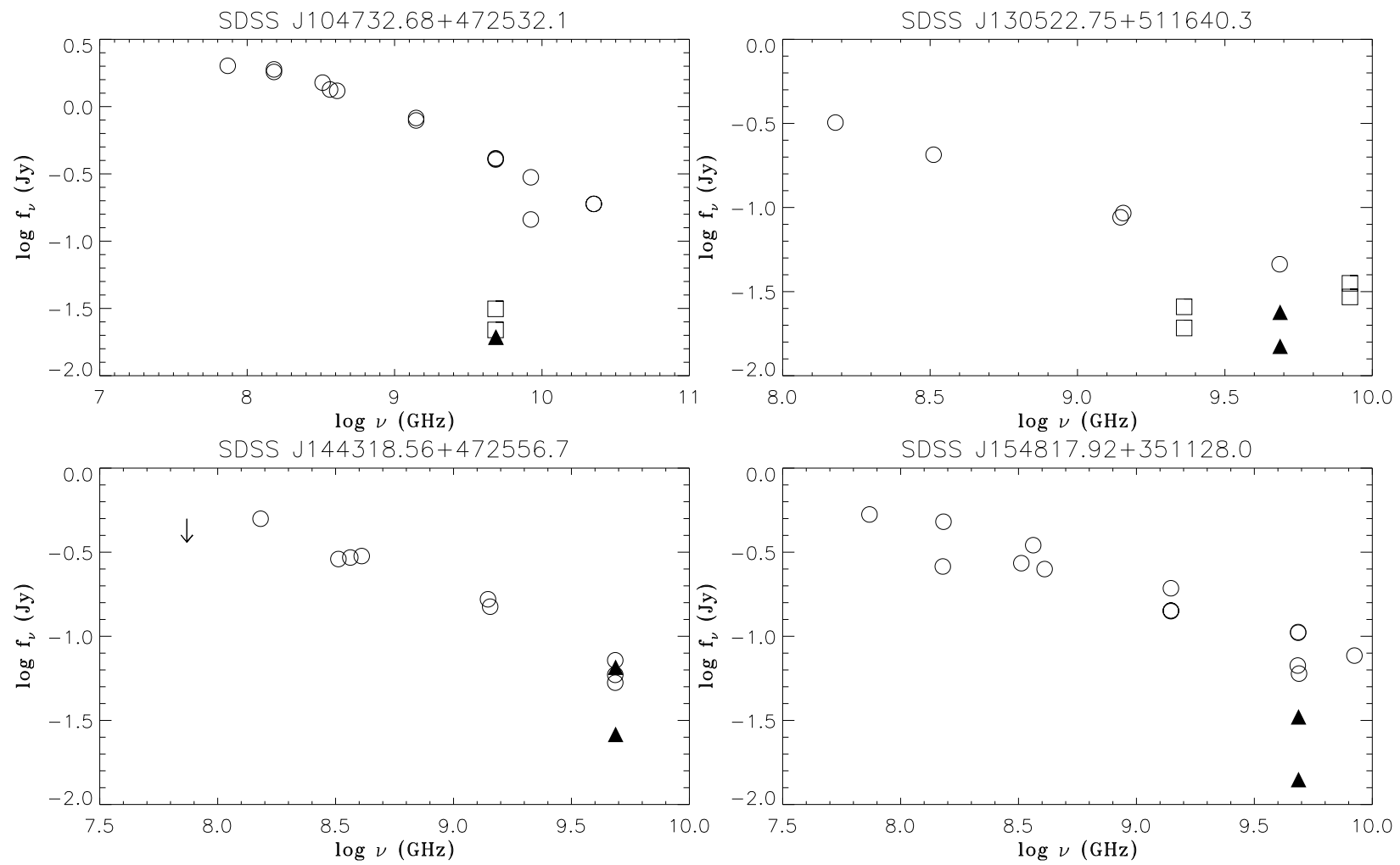

Fig. 17. - Radio spectra. The circles are based on NED data. The solid triangles are core and total VLBA flux densities from our measurements at $5 \mathrm{GHz}$. The rectangles are core and total VLBA flux densities from measurements on the archival data. The downward arrow is the upper limit of the flux density at $74 \mathrm{MHz}$. 
Table 1: Sample of Radio-loud NLS1 Galaxies

\begin{tabular}{lcccccccc}
\hline \hline Name & $z$ & $\begin{array}{c}f_{1.4 \mathrm{GHz}} \\
(\mathrm{mJy})\end{array}$ & $\begin{array}{c}f_{5 \mathrm{GHz}} \\
(\mathrm{mJy})\end{array}$ & $\alpha$ & $\log R$ & $\begin{array}{c}\mathrm{a} \\
(\operatorname{arcsec})\end{array}$ & $\begin{array}{c}\text { LS } \\
(\mathrm{kpc})\end{array}$ & $\begin{array}{c}\text { r.s. } \\
(1)\end{array}$ \\
\hline SDSS J081432.11+560956.6 & 0.509 & $69 / 60$ & $43)$ & -0.33 & 2.53 & 2.84 & 17.4 & $\mathrm{~F}$ \\
SDSS J085001.17+462600.5 & 0.523 & $21 / 16$ & & & 2.23 & 1.26 & 7.8 & $\mathrm{~S}$ \\
SDSS J090227.16+044309.6 & 0.532 & $153 / 157$ & 106 & -0.30 & 3.02 & 1.00 & 6.3 & $\mathrm{~F}$ \\
SDSS J095317.09+283601.5 & 0.657 & $45 / 43$ & & & 2.71 & 1.95 & 13.6 & $\mathrm{~S}^{a}$ \\
SDSS J103727.45+003635.6 & 0.595 & $27 / 28$ & & & 2.66 & 1.16 & 7.7 & $\mathrm{~F}^{a}$ \\
SDSS J104732.68+472532.1 & 0.798 & $734 / 789$ & 404 & -0.51 & 3.87 & 1.16 & 8.7 & $\mathrm{~S}$ \\
SDSS J111005.03+365336.3 & 0.630 & $19 / 23$ & & & 2.97 & 2.08 & 14.2 & $\mathrm{~S}$ \\
SDSS J113824.54+365327.1 & 0.356 & $13 / 12$ & & & 2.34 & 0.43 & 2.1 & $\mathrm{~S}$ \\
SDSS J124634.65+023809.0 & 0.362 & $37 / 36$ & 46 & 0.17 & 2.38 & 1.12 & 5.6 & $\mathrm{~F}$ \\
SDSS J130522.75+511640.3 & 0.785 & $84 / 87$ & 46 & -0.50 & 2.34 & 1.34 & 10.0 & $\mathrm{~S}$ \\
SDSS J142114.05+282452.8 & 0.538 & $48.7 / 45.4$ & 40 & -0.15 & 2.31 & 1.28 & 8.1 & $\mathrm{~F}$ \\
SDSS J144318.56+472556.7 & 0.703 & $165 / 166$ & 72 & -0.67 & 3.07 & 1.18 & 8.4 & $\mathrm{~S}$ \\
SDSS J154817.92+351128.0 & 0.478 & $141 / 141$ & 107 & -0.22 & 2.84 & 0.51 & 3.0 & $\mathrm{~F}$ \\
SDSS J163401.94+480940.2 & 0.494 & $8 / 14$ & & & 2.31 & 1.15 & 6.9 & $\mathrm{~F}$ \\
\hline
\end{tabular}

Notes. - Col. (1): source name; Col. (2) redshift; Col. (3): the FIRST and NVSS flux densities at 1.4 $\mathrm{GHz}$ (in the format of FIRST/NVSS); Col. (4): the flux density at $5 \mathrm{GHz}$ from the literature; Col. (5): the spectral index between 1.4 and $5 \mathrm{GHz}$ when available $\left(f_{\nu} \propto \nu^{\alpha}\right)$; Col. (6): the radio loudness defined as $f_{\nu}(1.4 \mathrm{GHz}) / f_{\nu}(4400 \AA)$; Col. (7): the major axis from FIRST; Col. (8): the upper limit on the source linear size calculated from the FIRST major axis; Col. (9): the radio spectrum based on low-resolution NED data (see Section 4.3): F - flat spectrum, S - steep spectrum, ${ }^{a}$ - uncertain (see text for details). 
Table 2. Results for the radio-loud NLS1 Galaxies

\begin{tabular}{|c|c|c|c|c|c|c|c|}
\hline $\begin{array}{l}\text { Name } \\
(1)\end{array}$ & $\begin{array}{c}\text { Comps. } \\
\text { (2) }\end{array}$ & $\begin{array}{c}S \\
(\mathrm{mJy}) \\
(3)\end{array}$ & $\begin{array}{c}r \\
(\mathrm{mas}) \\
(4)\end{array}$ & $\begin{array}{c}\theta \\
(\mathrm{deg}) \\
(5)\end{array}$ & $\begin{array}{c}a \\
(\mathrm{mas}) \\
(6)\end{array}$ & $\begin{array}{l}b / a \\
(7)\end{array}$ & $\begin{array}{c}\log T_{\mathrm{b}} \\
(\mathrm{K}) \\
(8)\end{array}$ \\
\hline SDSS J081432.11+560956.6 & $\mathrm{C}$ & $\begin{array}{c}19.3 \\
6.3\end{array}$ & & $\begin{array}{r}-89.4 \\
91.0\end{array}$ & $\begin{array}{l}0.41 \\
3.64\end{array}$ & $\begin{array}{l}1.00 \\
1.00\end{array}$ & 10.1 \\
\hline SDSS J085001.17+462600.5 & $\mathrm{C}$ & 11.0 & 0.08 & -74.4 & 0.43 & 1.00 & 9.8 \\
\hline SDSS J090227.16+044309.6 & $\mathrm{C}$ & $\begin{array}{c}49.7 \\
22.9 \\
21.5 \\
7.5\end{array}$ & $\begin{array}{l}0.26 \\
0.62 \\
2.62 \\
4.13\end{array}$ & $\begin{array}{r}-124.3 \\
53.3 \\
63.0 \\
54.9\end{array}$ & $\begin{array}{l}0.16 \\
0.58 \\
1.15 \\
0.73\end{array}$ & $\begin{array}{l}1.00 \\
1.00 \\
1.00 \\
1.00\end{array}$ & 11.4 \\
\hline SDSS J095317.09+283601.5 & $\mathrm{C}$ & $\begin{array}{c}12.7 \\
1.4\end{array}$ & $\begin{array}{l}0.03 \\
2.08\end{array}$ & $\begin{array}{l}88.1 \\
32.7\end{array}$ & $\begin{array}{l}0.63 \\
0.68\end{array}$ & $\begin{array}{l}1.00 \\
1.00\end{array}$ & 9.6 \\
\hline SDSS J103727.45+003635.6 & $\mathrm{C}$ & 21.9 & 0.04 & -156.7 & 0.59 & 0.25 & 10.5 \\
\hline SDSS J104732.68+472532.1 & $\mathrm{C}$ & 19.4 & 1.37 & 98.2 & 5.39 & 0.36 & 8.4 \\
\hline SDSS J111005.03+365336.3 & $\mathrm{C}$ & 8. & 0.13 & -7.7 & 0.44 & 0.50 & 10.0 \\
\hline SDSS J113824.54† & $\mathrm{C}$ & 9.5 & 0.06 & -43.3 & 0.77 & 0.52 & 9.5 \\
\hline SDSS J124634.65+023809.0 & $\mathrm{C}$ & 8.7 & 0.13 & 176.1 & 0.62 & 0.46 & 9.7 \\
\hline SDSS J130522.75+511640.3 & $\mathrm{C}$ & $\begin{array}{c}15.0 \\
8.9\end{array}$ & $\begin{array}{l}0.05 \\
5.97\end{array}$ & $\begin{array}{r}88.5 \\
-173.7\end{array}$ & $\begin{array}{l}0.20 \\
2.23\end{array}$ & $\begin{array}{l}0.85 \\
0.29\end{array}$ & 10.8 \\
\hline SDSS J142114.05+282452.8 & $\mathrm{C}$ & $\begin{array}{c}27.1 \\
5.8 \\
0.9\end{array}$ & $\begin{array}{l}0.10 \\
0.66 \\
3.45\end{array}$ & $\begin{array}{l}-76.8 \\
118.4 \\
126.6\end{array}$ & $\begin{array}{l}0.18 \\
0.44 \\
3.92\end{array}$ & $\begin{array}{l}1.00 \\
1.00 \\
0.34\end{array}$ & 11.0 \\
\hline SDSS J144318.56+472556.7 & $\mathrm{C}^{a}$ & $\begin{array}{c}26.1 \\
18.0 \\
5.5 \\
2.7 \\
3.6 \\
6.4 \\
3.3\end{array}$ & $\begin{array}{c}0.45 \\
0.99 \\
2.07 \\
3.43 \\
7.72 \\
11.19 \\
13.66\end{array}$ & $\begin{array}{r}-60.2 \\
124.4 \\
104.5 \\
102.9 \\
88.8 \\
99.9 \\
106.0\end{array}$ & $\begin{array}{l}0.39 \\
0.93 \\
0.21 \\
1.47 \\
2.85 \\
2.81 \\
2.95\end{array}$ & $\begin{array}{l}1.00 \\
1.00 \\
1.00 \\
1.00 \\
1.00 \\
1.00 \\
1.00\end{array}$ & 10.3 \\
\hline SDSS J154817.92+351128.0 & $\mathrm{C}$ & $\begin{array}{c}14.1 \\
4.5 \\
8.4\end{array}$ & $\begin{array}{c}0.04 \\
7.05 \\
19.52\end{array}$ & $\begin{array}{r}-103.6 \\
5.6 \\
10.6\end{array}$ & $\begin{array}{l}0.98 \\
1.65 \\
7.87\end{array}$ & $\begin{array}{l}0.21 \\
0.27 \\
0.40\end{array}$ & 9.9 \\
\hline
\end{tabular}


Table 2 - Continued

\begin{tabular}{|c|c|c|c|c|c|c|c|}
\hline $\begin{array}{c}\text { Name } \\
(1)\end{array}$ & $\begin{array}{c}\text { Comps. } \\
\text { (2) }\end{array}$ & $\begin{array}{c}S \\
(\mathrm{mJy}) \\
(3)\end{array}$ & $\begin{array}{c}r \\
(\mathrm{mas}) \\
(4)\end{array}$ & $\begin{array}{c}\theta \\
(\mathrm{deg}) \\
(5)\end{array}$ & $\begin{array}{c}a \\
(\mathrm{mas}) \\
(6)\end{array}$ & $\begin{array}{l}b / a \\
(7)\end{array}$ & $\begin{array}{c}\log T_{\mathrm{b}} \\
(\mathrm{K}) \\
(8)\end{array}$ \\
\hline SDSS J163401.94+480940.2 & $\mathrm{C}$ & $\begin{array}{l}6.2 \\
9.5\end{array}$ & $\begin{array}{c}59.30 \\
0.11\end{array}$ & $\begin{array}{r}26.2 \\
-152.9\end{array}$ & $\begin{array}{l}6.84 \\
0.29\end{array}$ & $\begin{array}{l}0.78 \\
1.00\end{array}$ & 10.1 \\
\hline
\end{tabular}

Col. (1): source name; Col. (2): components: $\mathrm{C}=\mathrm{core},{ }^{a}$ - core identification is uncertain (see text for details); Col. (3) flux density; Col. (4)-(5): component position, and its position angle; Col. (6): major axis; Col. (7): axial ratio; Col. (8): brightness temperature. 
Table 3. Archive data of Radio-loud NLS1 Galaxies

\begin{tabular}{|c|c|c|c|c|c|c|c|c|c|c|}
\hline $\begin{array}{c}\text { Name } \\
(1)\end{array}$ & $\begin{array}{l}\text { array } \\
(2)\end{array}$ & $\begin{array}{c}\text { obs. date } \\
\text { (3) }\end{array}$ & $\begin{array}{c}\nu \\
(\mathrm{GHz}) \\
(4)\end{array}$ & $\begin{array}{l}\text { Comps. } \\
\text { (5) }\end{array}$ & $\begin{array}{c}S \\
(\mathrm{mJy}) \\
(6)\end{array}$ & $\begin{array}{c}r \\
(\mathrm{mas}) \\
(7)\end{array}$ & $\begin{array}{c}\theta \\
(\mathrm{deg}) \\
(8)\end{array}$ & $\begin{array}{c}a \\
(\mathrm{mas}) \\
(9)\end{array}$ & $\begin{array}{l}b / a \\
(10)\end{array}$ & $\begin{array}{c}\log T_{\mathrm{b}} \\
(\mathrm{K}) \\
(11)\end{array}$ \\
\hline \multirow[t]{7}{*}{ SDSS J081432.11+560956.6 } & VLBA & 2010-Mar-23 & 2.3 & $\mathrm{C}$ & 23.3 & 0.11 & -76.0 & 0.14 & 1.00 & 11.8 \\
\hline & & & 2.3 & & 7.2 & 2.43 & 82.7 & 0.26 & 1.00 & \\
\hline & VLBA & 2006-May-31 & 5.0 & $\mathrm{C}$ & 30.7 & 0.09 & -106.7 & 0.47 & 0.50 & 10.5 \\
\hline & & & 5.0 & & 8.5 & 1.85 & 93.6 & 1.55 & 0.32 & \\
\hline & VLBA & 2010-Mar-23 & 8.4 & $\mathrm{C}$ & 29.3 & 0.03 & -88.0 & 0.06 & 1.00 & 11.5 \\
\hline & & & 8.4 & & 4.7 & 0.37 & 90.8 & 0.22 & 1.00 & \\
\hline & & & 8.4 & & 1.9 & 2.55 & 89.5 & 0.55 & 1.00 & \\
\hline \multirow[t]{6}{*}{ SDSS J104732.68+472532.1 } & VLBA & 2006-May-01 & 5.0 & $\mathrm{C}$ & 21.9 & 0.31 & -28.3 & 2.10 & 0.74 & $9.0_{0}$ \\
\hline & & & 5.0 & & 9.5 & 4.05 & 85.0 & 1.74 & 0.22 & $\infty$ \\
\hline & VLA & 1990-Feb-20 & 8.4 & $\mathrm{C}$ & 144.8 & 7.90 & 143.5 & 114.49 & 0.47 & \\
\hline & & & 8.4 & & 90.7 & 298.10 & -41.5 & 357.23 & 0.63 & \\
\hline & & & 8.4 & & 62.6 & 271.44 & 108.9 & 75.31 & 1.00 & \\
\hline & VLA & 2009-Sep-01 & 22.4 & & 189.1 & 17.25 & 112.2 & 442.07 & 0.38 & \\
\hline \multirow[t]{6}{*}{ SDSS J130522.75+511640.3 } & VLBA & 2011-Mar-15 & 2.3 & $\mathrm{C}$ & 19.2 & 0.36 & -10.3 & 0.27 & 1.00 & 11.2 \\
\hline & & & 2.3 & & 4.5 & 2.85 & -146.9 & 1.24 & 1.00 & \\
\hline & & & 2.3 & & 1.9 & 9.13 & -158.1 & 0.79 & 1.00 & \\
\hline & VLBA & 2011-Mar-15 & 8.4 & $\mathrm{C}$ & 29.4 & 0.00 & -22.6 & 0.09 & 1.00 & 11.3 \\
\hline & & & 8.4 & & 4.2 & 5.57 & -172.5 & 1.24 & 0.35 & \\
\hline & & & 8.4 & & 1.9 & 7.54 & -169.9 & 1.73 & 0.72 & \\
\hline \multirow[t]{2}{*}{ SDSS J142114.05+282452.8 } & VLBA & 2010-Mar-23 & 2.3 & $\mathrm{C}$ & 33.0 & 0.04 & 5.4 & 0.67 & 1.00 & 10.6 \\
\hline & & & 2.3 & & 2.3 & 6.90 & 138.9 & 1.01 & 1.00 & \\
\hline
\end{tabular}


Table 3-Continued

\begin{tabular}{|c|c|c|c|c|c|c|c|c|c|c|}
\hline $\begin{array}{l}\text { Name } \\
\text { (1) }\end{array}$ & $\begin{array}{l}\text { array } \\
(2)\end{array}$ & $\begin{array}{l}\text { obs. date } \\
\text { (3) }\end{array}$ & $\begin{array}{c}\nu \\
(\mathrm{GHz}) \\
(4)\end{array}$ & $\begin{array}{l}\text { Comps. } \\
\text { (5) }\end{array}$ & $\begin{array}{c}S \\
(\mathrm{mJy}) \\
(6)\end{array}$ & $\begin{array}{c}r \\
(\mathrm{mas}) \\
(7)\end{array}$ & $\begin{array}{c}\theta \\
(\mathrm{deg}) \\
(8)\end{array}$ & $\begin{array}{c}a \\
(\mathrm{mas}) \\
(9)\end{array}$ & $\begin{array}{l}b / a \\
(10)\end{array}$ & $\begin{array}{c}\log T_{\mathrm{b}} \\
(\mathrm{K}) \\
(11)\end{array}$ \\
\hline & VLBA & 2010-Mar-23 & 8.4 & $\mathrm{C}$ & 21.6 & 0.08 & 136.2 & 0.48 & 0.29 & 10.1 \\
\hline
\end{tabular}

-Col. (1): source name; Col. (2) radio telescope array; Col. (3) observational date; Col. (4): observing frequency; Col. (5): components: $\mathrm{C}=$ core; Col. (6): flux density; Col. (7)-(8): component position, and its position angle; Col. (9): major axis; Col. (10): axial ratio; Col. (11): brightness temperature. 